\title{
SHARP SOBOLEV INEQUALITIES INVOLVING BOUNDARY TERMS
}

\author{
Y.Y. LI AND M. ZHU
}

\section{Introduction}

Let $(M, g)$ be a compact Riemannian manifold of dimension $n(n \geq 3)$ with smooth boundary. In [LZ], we established some sharp trace inequality on $(M, g)$. In this paper we establish some sharp Sobolev inequalities using the method in [LZ].

For $n \geq 3$, it was shown by Aubin [Au1] and Talenti [T] that, for $p=2 n /(n-2)$,

$$
\frac{1}{S_{1}}=\inf \left\{\frac{\int_{\mathbb{R}^{n}}|\nabla u|^{2}}{\left(\int_{\mathbb{R}^{n}}|u|^{p}\right)^{2 / p}} \mid u \in L^{p}\left(\mathbb{R}^{n}\right) \backslash\{0\}, \nabla u \in L^{2}\left(\mathbb{R}^{n}\right)\right\},
$$

is achieved and the extremal functions are found. In particular,

$$
\frac{1}{S_{1}}=\pi n(n-2)(\Gamma(n / 2) / \Gamma(n))^{2 / n} .
$$

It follows that for any genuine open subset $\Omega$ of $\mathbb{R}^{n}$,

$$
\left(\int_{\Omega}|u|^{p}\right)^{2 / p}<S_{1} \int_{\Omega}|\nabla u|^{2}, \quad \forall u \in H_{0}^{1}(\Omega) \backslash\{0\},
$$

and, by even reflection,

$$
\left(\int_{\mathbb{R}_{+}^{n}}|u|^{p}\right)^{2 / p} \leq 2^{2 / n} S_{1} \int_{\mathbb{R}_{+}^{n}}|\nabla u|^{2}
$$

for all $u$ satisfying $u \in L^{p}\left(\mathbb{R}_{+}^{n}\right)$ and $\nabla u \in L^{2}\left(\mathbb{R}_{+}^{n}\right)$, where $\mathbb{R}_{+}^{n}=\left\{\left(x_{1}, \ldots, x_{n}\right)\right.$ $\left.\in \mathbb{R}^{n}: x_{n}>0\right\}$.

It was shown by P.L. Lions [Lio] that, for $q=2(n-1) /(n-2)$,

$$
\frac{1}{S}=\inf \left\{\frac{\int_{\mathbb{R}_{+}^{n}}|\nabla u|^{2}}{\left(\int_{\partial \mathbb{R}_{+}^{n}}|u|^{q}\right)^{2 / q}} \mid \nabla u \in L^{2}\left(\mathbb{R}_{+}^{n}\right), u \in L^{q}\left(\partial \mathbb{R}_{+}^{n}\right) \backslash\{0\}\right\},
$$

The first author is partially supported by the Alfred P. Sloan Foundation Research Fellowship, NSF grant DMS-9401815, and a Rutgers University Research Council Grant. 
is achieved. The extremal functions are found independently by Escobar [E2] and Beckner [B]. In particular

$$
\frac{1}{S}=\frac{n-2}{2} \sigma_{n}^{1 /(n-1)}
$$

where $\sigma_{n}$ denotes the volume of the unit sphere in $\mathbb{R}^{n}$.

Some related sharp inequalities on $\mathbb{R}_{+}^{n}$ are given by Brezis and Lieb [BrLi], Carlen and Loss [CaLo1-2]. Many related problems were also discussed in Trudinger [Tr], Moser [M], Lieb [Li1-2], Aubin [Au2], BrezisNirenberg [BrNi], Cherrier [Che1-2], Carleson-Chang [CarCh], Escobar [E1], Hamza [H], Beckner [B], Adimurthi and Yadava [AY], Hebey and Vaugon [HeV1-2], Hebey [He], and the references therein.

In this paper we establish the following result.

Theorem 0.1. For $n \geq 3$, let $(M, g)$ be a smooth $n$-dimensional compact Riemannian manifold with smooth boundary. Then a necessary and sufficient condition for the existence of some constant $A(M, g)>0$ so that

$$
\left(\int_{M}|u|^{p} d v_{g}\right)^{2 / p} \leq 2^{2 / n} S_{1} \int_{M}\left|\nabla_{g} u\right|^{2} d v_{g}+A(M, g) \int_{\partial M} u^{2} d s_{g}, \forall u \in H^{1}(M),
$$

holds is

$$
\left(\int_{M}|u|^{p} d v_{g}\right)^{2 / p}<2^{2 / n} S_{1} \int_{M}\left|\nabla_{g} u\right|^{2} d v_{g}, \quad \forall u \in H_{0}^{1}(M) \backslash\{0\},
$$

where $d v_{g}$ denotes the volume form of $g$, and $d s_{g}$ denotes the induced volume form on $\partial M$.

An easy consequence of Theorem 0.1 is, in view of (0.2), the following.

Corollary 0.1. For $n \geq 3$, let $\Omega \subset \mathbb{R}^{n}$ be a smooth bounded open set. Then there exists some $A=A(\Omega)>0$ such that

$$
\left(\int_{\Omega}|u|^{p}\right)^{2 / p} \leq 2^{2 / n} S_{1} \int_{\Omega}|\nabla u|^{2}+A(\Omega) \int_{\partial \Omega} u^{2}, \quad \forall u \in H^{1}(\Omega) .
$$

REMARK 0.1. One cannot expect (0.5) to hold for all compact manifolds with boundary, as shown by the following example.

Let $\varphi_{\epsilon} \subset C^{\infty}\left(\mathbb{R}^{n}\right)$ be some positive function satisfying

$$
\varphi_{\epsilon}(x)= \begin{cases}\epsilon^{-1}, & |x| \leq \sqrt{\epsilon} \\ 1, & |x| \geq 1 / 4\end{cases}
$$

Consider $g_{\epsilon}=\varphi_{\epsilon}^{2} g_{0}$, where $g_{0}$ is the flat metric on $\mathbb{R}^{n}$. On $\left(B_{1}, g_{\epsilon}\right)$, we take $u \in C_{c}^{\infty}\left(B_{1}\right)$ which is independent of $\epsilon$ and satisfies $u(x)=1$ for all 
$|x| \leq 1 / 2$. It is very easy to see that for $\epsilon>0$ small, $u$ satisfies

$$
\left(\int_{B_{1}}|u|^{p} d v_{g_{\epsilon}}\right)^{2 / p}>2^{2 / n} S_{1} \int_{B_{1}}\left|\nabla_{g_{\epsilon}} u\right|^{2} d v_{g_{\epsilon}} .
$$

Therefore (0.5) does not hold for $\left(B_{1}, g_{\epsilon}\right)$.

REMARK 0.2. In (0.5), the constant $2^{2 / n} S_{1}$ in front of $\int_{M}\left|\nabla_{g} u\right|^{2} d v_{g}$ is sharp. It cannot be replaced by any smaller numbers.

REMARK 0.3. In general, $\int_{\partial M} u^{2} d s_{g}$ in (0.5) cannot be replaced by $\int_{\partial M} u^{r} d s_{g}$ for $r<2$. For instance, this is the case for any bounded domain in $\mathbb{R}^{n}$ with the flat metric.

REMARK 0.4. Corollary 0.1 in the case $n \geq 5$ was established in [AY] by Adimurthi and Yadava. Our method is different.

As in [LZ], there are two main ingredients in our proof of the sufficient part of Theorem 0.1. One is to obtain, by using the Moser iteration technique, an appropriate pointwise upper bound for blow up minimum energy solutions to certain critical exponent equations with nonlinear Neumann boundary conditions. The other is a local balance checking via Pohozaev identity. The method is useful in treating similar problems. For instance, We also show the following.

Theorem 0.2. For $n \geq 3$, let $(M, g)$ be some smooth $n$-dimensional compact Riemannian manifold with smooth boundary. Then there exists some constant $A_{1}=A_{1}(M, g)>0$ such that for all $u \in H^{1}(M)$,

$$
\left(\int_{M}|u|^{p} d v_{g}\right)^{2 / p} \leq 2^{2 / n} S_{1} \int_{M}\left|\nabla_{g} u\right|^{2} d v_{g}+A_{1} \int_{M} u^{2} d v_{g}+A_{1} \int_{\partial M} u^{2} d s_{g}
$$

REMARK 0.5. In (0.8), the constant $2^{2 / n} S_{1}$ in front of $\int_{M}\left|\nabla_{g} u\right|^{2} d v_{g}$ is sharp. It cannot be replaced by any smaller numbers.

In the following, we outline the ideas of the proof of Theorem 0.1. The proof of the necessary part of Theorem 0.1 is rather short and is given at the beginning of section 1 . In section 1 we also derive some preliminary estimates with almost sharp constants in front of $\int_{M}\left|\nabla_{g} u\right|^{2} d v_{g}$. We begin to prove the sufficient part of Theorem 0.1 by a contradiction argument from section 2. Suppose there did not exist any positive constant $A(M, g)$ for which (0.5) holds under condition (0.6), we would find, for any $\alpha>0$, some positive function $u_{\alpha} \in H^{1}(M)$ such that 


$$
\begin{aligned}
\frac{\int_{M}\left|\nabla_{g} u_{\alpha}\right|^{2} d v_{g}+\alpha \int_{\partial M} u_{\alpha}^{2} d s_{g}}{\left(\int_{M} u_{\alpha}^{p} d v_{g}\right)^{2 / p}} & =\inf _{H^{1}(M) \backslash\{0\}} \frac{\int_{M}\left|\nabla_{g} u\right|^{2} d v_{g}+\alpha \int_{\partial M} u^{2} d s_{g}}{\left(\int_{M}|u|^{p} d v_{g}\right)^{2 / p}} \\
& <\frac{1}{2^{2 / n} S_{1}}
\end{aligned}
$$

Without loss of generality, we assume $\int_{M} u_{\alpha}^{p} d v_{g}=1$. Since $u_{\alpha}$ is an extremal, it satisfies the Euler-Lagrange equation (see (2.2)). We mainly work with this equation to derive a contradiction. Let $x_{\alpha} \in M$ be some maximum point of $u_{\alpha}$, we prove, utilizing the preliminary estimates in section 1 , that $u_{\alpha}\left(x_{\alpha}\right) \rightarrow \infty$ and $u_{\alpha}$ blows up at precisely one point. We then show that $u_{\alpha}$, after appropriate scaling, is close to $v$ in energy, where $v$ is given explicitly in (2.13). Equivalently, if we scale back $v$ appropriately and denote it by $\varphi_{\alpha}$, we know that $\left\|u_{\alpha}-\varphi_{\alpha}\right\|_{H^{1}(M)} \rightarrow 0$ as $\alpha \rightarrow \infty$. Since $u_{\alpha}$ satisfies equation (2.2), we can write down some Pohozaev type identity for $u_{\alpha}$ in $B_{r_{\alpha}}\left(x_{\alpha}\right)$, the geodesic ball of radius $r_{\alpha}$ and centered at $x_{\alpha}$. If we substitute $\varphi_{\alpha}$ for $u_{\alpha}$ in the identity, with $r_{\alpha} \rightarrow 0$ appropriately chosen (depending on $\alpha, u_{\alpha}\left(x_{\alpha}\right)$ ), we see that the identity is unbalanced. More specifically, the term in the identity which is induced by the boundary term $-\alpha u_{\alpha}$ in (2.2) is much larger than the rest of the terms. However we have $u_{\alpha}$ instead of $\varphi_{\alpha}$ in the identity. Therefore we need to obtain good enough estimates on the difference $u_{\alpha}-\varphi_{\alpha}$. The estimate $\left\|u_{\alpha}-\varphi_{\alpha}\right\|_{H^{1}(M)} \rightarrow 0$ is not enough. In section 2 we establish the following crucial pointwise upper bound estimate of $u_{\alpha}: u_{\alpha} \leq C \varphi_{\alpha}$ on $M$ for some constant $C$ independent of $\alpha$. This is given in Proposition 2.1 and is proved, as in [LZ], by using the Moser iteration technique. Once we have this pointwise upper bound of $u_{\alpha}$, we derive from standard elliptic theories appropriate estimates on both $\left|\nabla u_{\alpha}\right|$ and $\left|\nabla^{2} u_{\alpha}\right|$. In dimension $n \geq 4$, these estimates are good enough to carry out the balance checking via a Pohozaev type identity described above to reach a contradiction. For dimension $n=3$, we still need another estimate: $u_{\alpha} \geq C^{-1} \varphi_{\alpha}$ in $B_{r_{\alpha}}\left(x_{\alpha}\right)$. This pointwise lower bound of $u_{\alpha}$ is obtained, as in [LZ], by using the maximum principle. We complete the proof of the sufficient part of Theorem 0.1 in section 3 . Theorem 0.2 is proved in a similar way and we sketch the proof in section 4 .

Throughout this paper we always denote $p=2 n /(n-2), q=$ $2(n-1) /(n-2),\|f\|_{r, M}=\left(\int_{M}|f|^{r} d v_{g}\right)^{1 / r}, S_{1}$ to be the constant in (0.1), $C$ to be various positive constants. 


\section{Necessary Part of Theorem 0.1 and Preliminary Estimates}

We first establish the necessary part of Theorem 0.1.

Proof of the necessary part of Theorem 0.1. It follows from (0.5) that

$$
\left(\int_{M}|u|^{p} d v_{g}\right)^{2 / p} \leq 2^{2 / n} S_{1} \int_{M}\left|\nabla_{g} u\right|^{2} d v_{g}, \quad \forall u \in H_{0}^{1}(M) \backslash\{0\} .
$$

If (0.6) did not hold, there would exist some nonnegative function $u_{0} \in$ $H_{0}^{1}(M) \backslash\{0\}$ such that

$$
\int_{M}\left|u_{0}\right|^{p} d v_{g}=1 \quad \text { and } \quad 2^{2 / n} S_{1} \int_{M}\left|\nabla_{g} u_{0}\right|^{2} d v_{g}=1 .
$$

Since $u_{0}$ is a minimizer to $\left\{\int_{M}\left|\nabla_{g} u\right|^{2} d v_{g}: \int_{M}|u|^{p} d v_{g}=1, u \in H_{0}^{1}(M)\right\}$, there exists some constant $\lambda$ such that

$$
\int_{M} \nabla_{g} u_{0} \nabla_{g} \varphi=\lambda \int_{M} u_{0}^{p-1} \varphi, \quad \forall \varphi \in H_{0}^{1}(M) .
$$

Take $\varphi=u_{0}$ in the above, we have $\lambda=1 /\left(2^{2 / n} S_{1}\right)$. Therefore $u_{0}$ satisfies

$$
\begin{cases}-\Delta_{g} u_{0}=\frac{1}{2^{2 / n} S_{1}} u_{0}^{p-1}, & \text { in } M, \\ u_{0}=0, & \text { on } \partial M .\end{cases}
$$

Let $v_{0} \in H^{1}(M)$ with $\left.v_{0}\right|_{\partial M}=\partial_{g} u_{0} / \partial \nu$, where $\partial_{g} / \partial \nu$ denotes differentiation in the direction of the unit outer normal of $\partial M$ with respect to $g$. Set

$$
F(\epsilon)=\left(\int_{M}\left|u_{0}+\epsilon v_{0}\right|^{p}\right)^{2 / p}-2^{2 / n} S_{1} \int_{M}\left|\nabla_{g}\left(u_{0}+\epsilon v_{0}\right)\right|^{2} .
$$

It follows from (1.1), (1.2) and the Hopf Lemma that

$$
F(0)=0, \quad F^{\prime}(0)=-2^{\frac{2+n}{n}} S_{1} \int_{\partial M}\left(\frac{\partial_{g} u_{0}}{\partial \nu}\right)^{2}<0 .
$$

On the other hand, in view of (0.5), we have

$$
F(\epsilon) \leq A \int_{\partial M}\left(u_{0}+\epsilon v_{0}\right)^{2}=A \epsilon^{2} \int_{\partial M} v_{0}^{2} .
$$

The above two inequalities lead to a contradiction for $\epsilon<0$ and close to zero. This establishes the necessary part of Theorem 0.1 .

In the rest of this section, we establish some rough inequalities which will serve as a starting point in our blow up analysis of the minimum energy sequence. 
Proposition 1.1. For $n \geq 3$, let $(M, g)$ be a smooth $n$-dimensional compact Riemannian manifold with smooth boundary. Then for all $\epsilon>0$, there exists some constant $B(\epsilon)$ depending only on $\epsilon$ and $(M, g)$ such that

$$
\left(\int_{M}|u|^{p} d s_{g}\right)^{\frac{2}{p}} \leq\left(2^{\frac{2}{n}} S_{1}+\epsilon\right) \int_{M}\left|\nabla_{g} u\right|^{2} d v_{g}+B(\epsilon) \int_{M}|u|^{2} d v_{g}, \forall u \in H^{1}(M) .
$$

The above proposition is standard. Readers can find a proof on page 51 of Aubin [Au2]. We omit the details.

Proposition 1.2. For $n \geq 3$, let $(M, g)$ be a smooth $n$-dimensional compact Riemannian manifold with smooth boundary. Suppose

$$
\left(\int_{M}|u|^{p} d v_{g}\right)^{2 / p} \leq 2^{2 / n} S_{1} \int_{M}\left|\nabla_{g} u\right|^{2} d v_{g}, \quad \forall u \in H_{0}^{1}(M) \backslash\{0\} .
$$

Then for all $\epsilon>0$, there exists some constant $A(\epsilon)$ depending only on $\epsilon$ and $(M, g)$ such that for all $u \in H^{1}(M)$,

$$
\left(\int_{M}|u|^{p} d v_{g}\right)^{\frac{2}{p}} \leq\left(2^{\frac{2}{n}} S_{1}+\epsilon\right) \int_{M}\left|\nabla_{g} u\right|^{2} d v_{g}+A(\epsilon) \int_{\partial M} u^{2} d s_{g} .
$$

Proof. We prove it by a contradiction argument. Suppose the contrary of (1.4), there exists some constant $\delta>0$, such that for all $\alpha>0$,

$$
\xi_{\alpha}:=\inf _{H^{1}(M) \backslash\{0\}} \frac{\int_{M}\left|\nabla_{g} u\right|^{2} d v_{g}+\alpha \int_{\partial M} u^{2} d s_{g}}{\left(\int_{M}|u|^{p} d v_{g}\right)^{2 / p}} \leq \frac{1}{2^{2 / n} S_{1}}-\delta .
$$

Due to (1.5), we can show as in [LZ] (see the proof of proposition 1.2 there) that there exists some nonnegative function $u_{\alpha} \in H^{1}(M)$ satisfying

$$
\xi_{\alpha}=\int_{M}\left|\nabla_{g} u_{\alpha}\right|^{2} d v_{g}+\alpha \int_{\partial M} u_{\alpha}^{2} d s_{g}, \quad \int_{M} u_{\alpha}^{p} d v_{g}=1 .
$$

Notice $\left\|u_{\alpha}\right\|_{H^{1}} \leq C$, there exists $\bar{u} \in H^{1}(M)$ such that $u_{\alpha} \rightarrow \bar{u}$ in $H^{1}(M)$. Since $\left\|u_{\alpha}\right\|_{2, \partial M} \rightarrow 0$ as $\alpha \rightarrow \infty, \bar{u} \in H_{0}^{1}(M)$. It is easy to see that

$$
\int_{M} u_{\alpha}^{p}-\int_{M}\left|u_{\alpha}-\bar{u}\right|^{p}-\int_{M} \bar{u}^{p} \rightarrow 0, \quad \text { as } \alpha \rightarrow \infty,
$$

and, in view of (1.6),

$$
\int_{M}\left|u_{\alpha}-\bar{u}\right|^{p} \leq 1+o(1), \quad \int_{M} \bar{u}^{p} \leq 1,
$$

where and throughout this section $o(1) \rightarrow 0$ as $\alpha \rightarrow \infty$. Also, from Proposition 1.1 we know that for $\delta / 2$ there exists a constant $B=B(\delta)$, such 
that

$$
\begin{gathered}
\left(\frac{1}{2^{2 / n} S_{1}}-\frac{\delta}{2}\right)\left(\int_{M}|\varphi|^{p} d v_{g}\right)^{2 / p} \leq \int_{M}\left|\nabla_{g} \varphi\right|^{2} d v_{g}+B(\delta) \int_{M} \varphi^{2} d v_{g} \\
\forall \varphi \in H^{1}(M)
\end{gathered}
$$

It follows from (1.9), the compact embedding of $H^{1}$ to $L^{2},(1.5),(1.7)$, and (1.8) that

$$
\begin{aligned}
\xi_{\alpha} & =\int_{M}\left|\nabla_{g} u_{\alpha}\right|^{2}+\alpha \int_{\partial M} u_{\alpha}^{2} \\
& =\int_{M}\left|\nabla_{g}\left(u_{\alpha}-\bar{u}\right)\right|^{2}+\int_{M}\left|\nabla_{g} \bar{u}\right|^{2}+\alpha \int_{\partial M} u_{\alpha}^{2}+o(1) \\
& \geq\left(\frac{1}{2^{2 / n} S_{1}}-\frac{\delta}{2}\right)\left(\int_{M}\left|u_{\alpha}-\bar{u}\right|^{p}\right)^{2 / p}+\xi_{\alpha}\left(\int_{M} \bar{u}^{p}\right)^{2 / p}+\alpha \int_{\partial M} u_{\alpha}^{2}+o(1) \\
& \geq\left(\frac{1}{2^{2 / n} S_{1}}-\frac{\delta}{2}-\xi_{\alpha}\right) \int_{M}\left|u_{\alpha}-\bar{u}\right|^{p}+\xi_{\alpha}+\alpha \int_{\partial M} u_{\alpha}^{2}+o(1) .
\end{aligned}
$$

Therefore, $\left\|u_{\alpha}-\bar{u}\right\|_{p, M} \rightarrow 0$. From this, we derive

$$
\frac{\left\|\nabla_{g} \bar{u}\right\|_{2, M}^{2}}{\|\bar{u}\|_{p, M}^{2}} \leq \frac{1}{2^{2 / n} S_{1}}-\delta
$$

which contradicts to the assumption of Proposition 1.2. This establishes Proposition 1.2.

The following lemma is due to Brezis and Lieb [BrLi].

LEMMA 1.1. Let $\Omega \subset \mathbb{R}^{n}$ be a bounded domain with smooth boundary, equipped with the standard Euclidean metric. Then there exists $A(\Omega)>0$ such that

$$
\|u\|_{p, \Omega} \leq S_{1}^{1 / 2}\|\nabla u\|_{2, \Omega}+A(\Omega)\|u\|_{q, \partial \Omega}, \quad u \in H^{1}(\Omega),
$$

where and throughout this paper, we assume $q=2(n-1) /(n-2)$.

Using Lemma 1.1 and Young's inequality, one easily sees that for any $\epsilon>0$,

$\|u\|_{p, \Omega}^{2} \leq\left(S_{1}+\epsilon\right)\|\nabla u\|_{2, \Omega}^{2}+\left(A^{2}(\Omega)+\frac{2 S_{1}^{1 / 2} A(\Omega)}{\epsilon}\right)\|u\|_{q, \partial \Omega}^{2}, \quad u \in H^{1}(\Omega)$.

In turn, we obtain, using the partition of unit, the following.

Proposition 1.3. For $n \geq 3$, let $(M, g)$ be a smooth $n$-dimensional compact Riemannian manifold with smooth boundary. Then for any $\epsilon>0$, there exists some constant $D(\epsilon)$ depending on $\epsilon$ such that for all $u \in H^{1}(M)$,

$$
\|u\|_{p, M}^{2} \leq\left(S_{1}+\epsilon\right)\left\|\nabla_{g} u\right\|_{2, M}^{2}+D(\epsilon)\|u\|_{q, \partial M}^{2}+D(\epsilon)\|u\|_{2, M}^{2} .
$$




\section{Asymptotic Analysis}

From now on we prove the sufficient part of Theorem 0.1. Namely, we establish (0.5) under the assumption (0.6). In the next two sections, we always assume (0.6). We argue by contradiction. Suppose not, we have, for all $\alpha \geq 1$,

$$
\xi_{\alpha}<\frac{1}{2^{2 / n} S_{1}}
$$

where $\xi_{\alpha}$ is defined in (1.5).

As explained in section 1 , there exists some nonnegative function $u_{\alpha} \in$ $H^{1}(M)$ satisfying (1.6). It follows that $u_{\alpha}$ satisfies

$$
\begin{cases}-\Delta_{g} u_{\alpha}=\xi_{\alpha} u_{\alpha}^{p-1} & \text { in } M, \\ \frac{\partial_{g} u_{\alpha}}{\partial \nu}=-\alpha u_{\alpha} & \text { on } \partial M,\end{cases}
$$

where, as always, $\partial_{g} / \partial \nu$ denotes differentiation in the direction of the unit outer normal of $\partial M$ with respect to $g$.

In this section, we establish the main estimates of $u_{\alpha}$. The crucial estimate is the pointwise upper bound estimate which we obtain in Proposition 2.1 through the Moser iteration technique.

For any $\epsilon>0$, it follows from (1.6), (2.1) and Proposition 1.2 that there exists some $A(\epsilon)$ such that

$$
\begin{aligned}
1+\frac{\epsilon}{2^{2 / n} S_{1}} & >\left(2^{2 / n} S_{1}+\epsilon\right) \xi_{\alpha} \\
& =\left(2^{2 / n} S_{1}+\epsilon\right)\left\|\nabla_{g} u_{\alpha}\right\|_{2, M}^{2}+\alpha\left(2^{2 / n} S_{1}+\epsilon\right)\left\|u_{\alpha}\right\|_{2, \partial M}^{2} \\
& \geq\left(\int_{M}\left|u_{\alpha}\right|^{p}\right)^{2 / p}+\left[\alpha\left(2^{2 / n} S_{1}+\epsilon\right)-A(\epsilon)\right]\left\|u_{\alpha}\right\|_{2, \partial M}^{2} \\
& =1+\left[\alpha\left(2^{2 / n} S_{1}+\epsilon\right)-A(\epsilon)\right]\left\|u_{\alpha}\right\|_{2, \partial M}^{2} .
\end{aligned}
$$

Thus

$$
\begin{aligned}
& 1+\frac{\epsilon}{2^{2 / n} S_{1}} \geq\left(2^{2 / n} S_{1}+\epsilon\right) \limsup _{\alpha \rightarrow \infty} \xi_{\alpha} \geq 1+\left(2^{2 / n} S_{1}+\epsilon\right) \limsup _{\alpha \rightarrow \infty} \alpha\left\|u_{\alpha}\right\|_{2, \partial M}^{2}, \\
& 1+\frac{\epsilon}{2^{2 / n} S_{1}} \geq\left(2^{2 / n} S_{1}+\epsilon\right) \liminf _{\alpha \rightarrow \infty} \xi_{\alpha} \geq 1+\left(2^{2 / n} S_{1}+\epsilon\right) \liminf _{\alpha \rightarrow \infty} \alpha\left\|u_{\alpha}\right\|_{2, \partial M}^{2} .
\end{aligned}
$$

Send $\epsilon \rightarrow 0$, we have

$$
\begin{aligned}
& \lim _{\alpha \rightarrow \infty} \xi_{\alpha}=\frac{1}{2^{2 / n} S_{1}}, \\
& \lim _{\alpha \rightarrow \infty} \alpha\left\|u_{\alpha}\right\|_{2, \partial M}^{2}=0 .
\end{aligned}
$$

It follows from Cherrier [Che1] that $u_{\alpha}$ is smooth up to boundary. Let $x_{\alpha}$ be some maximum point of $u_{\alpha}$, we set $\mu_{\alpha}=u_{\alpha}^{-2 /(n-2)}\left(x_{\alpha}\right)$. 
LEMMA 2.1.

$$
\lim _{\alpha \rightarrow \infty} \alpha \mu_{\alpha}=0
$$

Proof. We first claim

$$
\liminf _{\alpha \rightarrow \infty}\left\|u_{\alpha}\right\|_{q, \partial M}>0 .
$$

If the claim were false, i.e., $\left\|u_{\alpha}\right\|_{q, \partial M} \rightarrow 0$ along a subsequence $\alpha \rightarrow \infty$, then, in view of (1.6) and (2.1), there would exist $\bar{u} \in H_{0}^{1}(M)$ such that $u_{\alpha}$ weakly converges to $\bar{u}$. It is easy to check that $\bar{u}, u_{\alpha}$ satisfy (1.7) and (1.8). Therefore, it follows from Proposition 1.3 applied to $u_{\alpha}-\bar{u}$, the compact embedding of $H^{1}(M)$ to $L^{2}(M)$, the definition of $\xi_{\alpha},(1.7)$ and (1.8) that for any $\epsilon>0$

$$
\begin{aligned}
\xi_{\alpha} & =\int_{M}\left|\nabla_{g} u_{\alpha}\right|^{2}+\alpha \int_{\partial M} u_{\alpha}^{2} \\
& =\int_{M}\left|\nabla_{g}\left(u_{\alpha}-\bar{u}\right)\right|^{2}+\int_{M}\left|\nabla_{g} \bar{u}\right|^{2}+\alpha \int_{\partial M} u_{\alpha}^{2}+o(1) \\
& \geq\left(1 / S_{1}-\epsilon\right)\left(\int_{M}\left|u_{\alpha}-\bar{u}\right|^{p}\right)^{2 / p}+\xi_{\alpha}\left(\int_{M} \bar{u}^{p}\right)^{2 / p}+\alpha \int_{\partial M} u_{\alpha}^{2}+o(1) \\
& \geq\left(1 / S_{1}-\epsilon-\xi_{\alpha}\right) \int_{M}\left|u_{\alpha}-\bar{u}\right|^{p}+\xi_{\alpha}+\alpha \int_{\partial M} u_{\alpha}^{2}+o(1),
\end{aligned}
$$

where $o(1) \rightarrow 0$ as $\alpha \rightarrow \infty$. Take $\epsilon$ small enough, we derive by using (2.1) that $\left\|u_{\alpha}-\bar{u}\right\|_{p, M} \rightarrow 0$. In particular, in view of (1.6), $\int_{M} \bar{u}^{p}=1$. From this and (2.1), we have,

$$
\left(\int_{M}|\bar{u}|^{p} d v_{g}\right)^{2 / p} \geq 2^{2 / n} S_{1} \int_{M}\left|\nabla_{g} \bar{u}\right|^{2} d v_{g}
$$

which contradicts (0.6). This establishes (2.6).

It follows from (2.6), the definition of $\mu_{\alpha}$, and (2.4) that as $\alpha \rightarrow \infty$,

$$
\alpha \mu_{\alpha} \leq C \alpha \mu_{\alpha} \int_{\partial M} u_{\alpha}^{q} d s_{g} \leq C \alpha \int_{\partial M} u_{\alpha}^{2} \rightarrow 0 .
$$

We complete the proof of Lemma 2.1.

Let $\left(y^{1}, \ldots, y^{n-1}, y^{n}\right)$ denote some geodesic normal coordinates given by the exponential map $\exp _{x_{\alpha}}$. In this coordinate system, the metric $g$ is given by $g_{i j}(y) d y^{i} d y^{j}$. For suitably small $\delta_{1}>0$ (independent of $\alpha$ ), we define $v_{\alpha}$ in a neighborhood of $z=0$ by

$$
v_{\alpha}(z)=u_{\alpha}^{-1}\left(x_{\alpha}\right) u_{\alpha}\left(\exp _{x_{\alpha}}\left(\mu_{\alpha} z\right)\right), \quad z \in O_{\alpha} \subset \mathbb{R}^{n},
$$

where

$$
O_{\alpha}=\left\{z \in \mathbb{R}^{n}:|z|<\delta_{1} / \mu_{\alpha}, \exp _{x_{\alpha}}\left(\mu_{\alpha} z\right) \in M\right\}
$$


We write $\partial O_{\alpha}=\Gamma_{\alpha}^{1} \cup \Gamma_{\alpha}^{2}$, where

$$
\Gamma_{\alpha}^{1}=\left\{z \in \partial O_{\alpha}: \exp _{x_{\alpha}}\left(\mu_{\alpha} z\right) \in \partial M\right\}, \Gamma_{\alpha}^{2}=\left\{z \in \partial O_{\alpha}: \exp _{x_{\alpha}}\left(\mu_{\alpha} z\right) \in M\right\} .
$$

It follows from (2.2) that $v_{\alpha}$ satisfies

$$
\begin{cases}-\Delta_{g_{\alpha}} v_{\alpha}=\xi_{\alpha} v_{\alpha}^{p-1}, & \text { in } O_{\alpha}, \\ \frac{\partial_{g_{\alpha}} v_{\alpha}}{\partial \nu}=-\alpha \mu_{\alpha} v_{\alpha}, & \text { on } \Gamma_{\alpha}^{1}, \\ v_{\alpha}(0)=1, \quad 0 \leq v_{\alpha} \leq 1, & \end{cases}
$$

where $g_{\alpha}$ denotes the metric on $O_{\alpha}$ given by $g_{\alpha}=g_{i j}\left(\mu_{\alpha} z\right) d z^{i} d z^{j}$. It follows from (2.8), Lemma 2.1 and standard elliptic estimates that for all $R>1$,

$$
\left\|v_{\alpha}\right\|_{C^{3}\left(B_{R} \cap \bar{O}_{\alpha}\right)} \leq C(R), \quad \forall \alpha \geq 1 .
$$

Let $\lim _{\alpha \rightarrow \infty} \operatorname{dist}\left(x_{\alpha}, \partial M\right) / \mu_{\alpha}=T \in[0, \infty]$. Therefore $v_{\alpha} \rightarrow v$ in $C^{3}\left(\overline{O_{\alpha}} \cap B_{R}(0)\right)$ for all $R>1$, where $v$ satisfies

$$
\begin{cases}-\Delta v=\frac{1}{2^{2 / n} S_{1}} v^{p-1} & \text { in } \mathbb{R}_{-T}^{n}, \\ \frac{\partial v}{\partial \nu}=0 & \text { on } \partial \mathbb{R}_{-T}^{n}, \\ v(0)=1, \quad 0 \leq v(x) \leq 1, & \end{cases}
$$

where $\mathbb{R}_{-T}^{n}=\left\{z=\left(z^{\prime}, z_{n}\right): z_{n}>-T\right\}$ for $0 \leq T<\infty$; for $T=\infty$, $\mathbb{R}_{-\infty}^{n}=\mathbb{R}^{n}$ and there is no boundary condition in the above.

LEMma 2.2. $T=0$.

Proof. We prove it by a contradiction argument. If $0<T<\infty$, then after making an even reflection of $v$, we obtain a positive solution of $-\Delta v=$ $2^{-2 / n} S_{1}^{-1} v^{p-1}$ in $\mathbb{R}^{n}$ with two local maximum points. This is impossible due to the results of Gidas-Ni-Nirenberg [GNNi] or Caffarelli-Gidas-Spruck [CGS]. If $T=\infty$, we know from [CGS] the explicit form of $v$ which yields

$$
\int_{\mathbb{R}^{n}} v^{p}=2 .
$$

The above can also be deduced from some well known results. For the reader's convenience, we include it here. We know from the explicit expression of $v$ given by [CGS] that $v$ is an extremal function for the Sobolev inequality, i.e.,

$$
\frac{\int_{\mathbb{R}^{n}}|\nabla v|^{2}}{\left(\int_{\mathbb{R}^{n}} v^{p}\right)^{2 / p}}=\frac{1}{S_{1}} .
$$

Multiplying the equation of $v$ by $v$ and integrating by parts, we obtain, in view of the explicit expression of $v$ given by [CGS], that

$$
\int_{\mathbb{R}^{n}}|\nabla v|^{2}=\frac{1}{2^{2 / n} S_{1}} \int_{\mathbb{R}^{n}} v^{p}
$$


Equality (2.11) follows immediately from the above two identities.

However for all $R>0$,

Sending $\alpha$ to $\infty$, we have

$$
\int_{B_{R}(0)} v_{\alpha}^{p} d v_{g_{\alpha}}=\int_{B_{\mu_{\alpha} R}\left(x_{\alpha}\right)} u_{\alpha}^{p} d v_{g} \leq 1
$$

$$
\int_{B_{R}(0)} v^{p} \leq 1
$$

which violates (2.11). We have thus established Lemma 2.2.

It follows from Lemma 2.2 that

$$
\lim _{\alpha \rightarrow \infty} \operatorname{dist}\left(x_{\alpha}, \partial M\right) / \mu_{\alpha}=0, v_{\alpha} \rightarrow v \text { in } C^{3}\left(\overline{O_{\alpha}} \cap B_{R}(0)\right) \text { for all } R>1 \text {, }
$$

where

$$
v(x)=\left(\frac{1}{1+c(n)|x|^{2}}\right)^{(n-2) / 2},
$$

and $c(n)=1 /\left(2^{2 / n}(n-2) n S_{1}\right)$. A direct calculation, or similar argument as above, yields

$$
\int_{\mathbb{R}_{+}^{n}} v^{p}=1
$$

Recall that the conformal Laplacian operator $L_{g}$ and the conformal boundary operator $B_{g}$ corresponding to $g$ are given by

$$
\left\{\begin{array}{l}
L_{g} \psi=\Delta_{g} \psi-a(n) R_{g} \psi \\
B_{g} \psi=\frac{\partial_{g} \psi}{\partial \nu}+b(n) H_{g} \psi
\end{array}\right.
$$

where $a(n)=\frac{n-2}{4(n-1)}, b(n)=\frac{n-2}{2}, R_{g}$ is the scalar curvature of $M$, and $H_{g}$ is the mean curvature of $\partial M$ with respect to the inner normal of $\partial M$ (e.g., the unit ball in $\mathbb{R}^{n}$ has positive mean curvature).

Let $\hat{g}=\varphi^{4 /(n-2)} g$ for some positive function $\varphi$ being chosen later, then

$$
\begin{cases}L_{\hat{g}}(\psi / \varphi)=\varphi^{-(n+2) /(n-2)} L_{g}(\psi) & \text { in } M, \\ B_{\hat{g}}(\psi / \varphi)=\varphi^{-n /(n-2)} B_{g}(\psi) & \text { on } \partial M\end{cases}
$$

Rewrite (2.2) as

$$
\begin{cases}-\Delta_{g} u_{\alpha}=\xi_{\alpha} u_{\alpha}^{p-1} & \text { in } M \\ \frac{\partial_{g} u_{\alpha}}{\partial \nu}+b(n) H_{g} u_{\alpha}=-\alpha u_{\alpha}+b(n) H_{g} u_{\alpha} & \text { on } \partial M .\end{cases}
$$

Let $\psi=u_{\alpha}$ in (2.16) and write $w_{\alpha}=u_{\alpha} / \varphi$, we have

$$
\begin{cases}\Delta_{g} u_{\alpha}-a(n) R_{g} u_{\alpha}=\varphi^{(n+2) /(n-2)}\left(\Delta_{\hat{g}} w_{\alpha}-a(n) R_{\hat{g}} w_{\alpha}\right) & \text { in } M, \\ \frac{\partial_{g} u_{\alpha}}{\partial \nu}+b(n) H_{g} u_{\alpha}=\varphi^{n /(n-2)}\left(\frac{\partial_{\hat{g}} w_{\alpha}}{\partial \nu}+b(n) H_{\hat{g}} w_{\alpha}\right) & \text { on } \partial M .\end{cases}
$$


Let $\psi=\varphi$ in (2.16), we get

$$
\begin{cases}-a(n) R_{\hat{g}} \varphi^{\frac{n+2}{n-2}}=\Delta_{g} \varphi-a(n) R_{g} \varphi & \text { in } M, \\ +b(n) H_{\hat{g}} \varphi^{n / n-2}=\frac{\partial_{g} \varphi}{\partial \nu}+b(n) H_{g} \varphi & \text { on } \partial M .\end{cases}
$$

Combining (2.17), (2.18) and (2.19), we reach the following equation

$$
\begin{cases}-\Delta_{\hat{g}} w_{\alpha}=\xi_{\alpha} w_{\alpha}^{p-1}+\left(\Delta_{g} \varphi / \varphi^{\frac{n+2}{n-2}}\right) w_{\alpha} & \text { in } M \\ \frac{\partial_{\hat{g}} w_{\alpha}}{\partial \nu}=-\left(\alpha / \varphi^{2 /(n-2)}+\frac{\partial_{g} \varphi}{\partial \nu} / \varphi^{n /(n-2)}\right) w_{\alpha} & \text { on } \partial M\end{cases}
$$

We will choose appropriate $\varphi=\varphi_{\alpha}$ to simplify (2.20) and then apply the Moser iteration technique to show that $w_{\alpha}$ is bounded above by some constant independent of $\alpha$. Without loss of generality, we assume $(M, g)$ is a smooth bounded open set of a slightly larger Riemannian manifold $(\widetilde{M}, g)$. Let $Q_{\alpha}$ be the closest point on $\partial M$ to $x_{\alpha}, \gamma$ be the geodesic in $\widetilde{M}$ with $\gamma(0)=Q_{\alpha}, \gamma^{\prime}(0)=\nu$. Set $P_{\alpha}=\gamma\left(t_{\alpha} \mu_{\alpha}\right)$ with $t_{\alpha}=(n-2) / \xi_{\alpha}$. Let $\left(y^{1}, \ldots, y^{n-1}, y^{n}\right)$ be some geodesic normal coordinate system of $T_{P_{\alpha}} \widetilde{M}$, with $\partial / \partial y^{n}=-\gamma^{\prime}\left(t_{\alpha} \mu_{\alpha}\right), \exp _{P_{\alpha}}: T_{P_{\alpha}} \widetilde{M} \rightarrow \widetilde{M}$ denoting the exponential map, $g_{i j}(y)=\left\langle\partial / \partial y^{i}, \partial / \partial y^{j}\right\rangle$ denoting the metric of $\widetilde{M}$, with $g_{i j}(0)=\delta_{i j}$, $\Gamma_{i j}^{k}(0)=0$, where $\Gamma_{i j}^{k}$ is the Christoffel symbol. We define $G_{P_{\alpha}}$ by

$$
\begin{cases}-\Delta_{g} G_{P_{\alpha}}=n(n-2) \omega_{n} \delta_{P_{\alpha}} & \text { in } \widetilde{M}, \\ G_{P_{\alpha}}=0 & \text { on } \partial \widetilde{M},\end{cases}
$$

where $\omega_{n}$ is the volume of the unit ball in $\mathbb{R}^{n}$. It follows from Appendix B in [LZ] that

where $E(y)$ satisfies

$$
G_{P_{\alpha}} \circ \exp _{P_{\alpha}}(y)=|y|^{2-n}+E(y),
$$

$$
|y|^{n-3}|E(y)|+|y|^{n-2}\left|\nabla_{g} E(y)\right| \leq C\left(\delta_{1}\right), \quad \forall|y| \leq \delta_{1} .
$$

Define $\varphi_{\alpha}: \bar{M} \rightarrow \mathbb{R}$ by

$$
\varphi_{\alpha}=t_{\alpha}^{n-2} \mu_{\alpha}^{(n-2) / 2} G_{P_{\alpha}}
$$

Clearly, $\varphi_{\alpha}$ satisfies

$$
-\Delta_{g} \varphi_{\alpha}=0 \text { in } M .
$$

The following lemma was established in [LZ] (Lemma 2.5 there).

Lemma 2.3. For $\alpha$ large,

$$
\alpha / \varphi_{\alpha}^{2 /(n-2)}+\frac{\partial_{g} \varphi_{\alpha}}{\partial \nu} / \varphi_{\alpha}^{n /(n-2)} \geq 0 \text { on } \partial M .
$$


From Lemma 2.3 and $(2.20)$, we know that for $\hat{g}=\varphi_{\alpha}^{4 /(n-2)} g, w_{\alpha}=$ $u_{\alpha} / \varphi_{\alpha}$, we have, for large $\alpha$,

$$
\begin{cases}-\Delta_{\hat{g}} w_{\alpha}=\xi_{\alpha} w_{\alpha}^{p-1} & \text { in } M \\ \frac{\partial_{\hat{g}} w_{\alpha}}{\partial \nu} \leq 0 & \text { on } \partial M\end{cases}
$$

LEMMA 2.4 .

$$
\lim _{\alpha \rightarrow \infty} \int_{O_{\alpha}}\left|v_{\alpha}-v\right|^{p}=0
$$

where $v$ is given by (2.13) and $O_{\alpha}$ is given by (2.7).

Proof. Using (2.12) and the fact $O_{\alpha}$ tends to $\mathbb{R}_{+}^{n}$, we have for all $R>1$,

$$
\lim _{\alpha \rightarrow \infty}\left\|v_{\alpha}-v\right\|_{L^{p}\left(O_{\alpha} \cap B_{R}(0)\right)}=0 .
$$

Consequently, in view of (2.14),

$$
\lim _{\alpha \rightarrow \infty}\left\|v_{\alpha}\right\|_{L^{p}\left(O_{\alpha} \cap B_{R}(0)\right)}=1+o_{R}(1),
$$

where $\circ_{R}(1)$ tends to zero as $R \rightarrow \infty$.

We see easily from (1.6) that $\left\|v_{\alpha}\right\|_{L^{p}\left(O_{\alpha}\right)} \leq 1$. Therefore, in view of (2.26), we have

$$
\limsup _{\alpha \rightarrow \infty}\left\|v_{\alpha}-v\right\|_{L^{p}\left(O_{\alpha} \backslash B_{R}(0)\right)}=o_{R}(1) .
$$

Lemma 2.4 follows from (2.25) and (2.27).

Now we are ready to prove the following crucial estimate.

Proposition 2.1. There exists some constant $C$ independent of $\alpha$ such that for all $\alpha \geq 1$

$$
u_{\alpha} / \varphi_{\alpha} \leq C \text { for } x \in \bar{M} .
$$

Proof. We only need to show (2.28) holds for $\alpha$ large, thus, without loss of generality, we assume $w_{\alpha}=u_{\alpha} / \varphi_{\alpha}$ satisfies (2.23).

Let $\eta$ be some smooth cutoff function. Multiplying (2.23) by $w_{\alpha}^{k} \eta^{2}$ for $k>1$ and integrating by parts, we obtain

$$
\int_{M} \nabla_{\hat{g}} w_{\alpha} \cdot \nabla_{\hat{g}}\left(w_{\alpha}^{k} \eta^{2}\right) d v_{\hat{g}} \leq \xi_{\alpha} \int_{M} w_{\alpha}^{p-1+k} \eta^{2} d v_{\hat{g}}
$$

Direct computation yields

$$
\begin{aligned}
\int_{M} \nabla_{\hat{g}} w_{\alpha} \cdot \nabla_{\hat{g}}\left(w_{\alpha}^{k} \eta^{2}\right) d v_{\hat{g}} & \\
= & \frac{4 k}{(k+1)^{2}} \int_{M}\left|\nabla_{\hat{g}}\left(w_{\alpha}^{k+1 / 2} \eta\right)\right|^{2} d v_{\hat{g}}+\frac{k-1}{(k+1)^{2}} \int_{M} w_{\alpha}^{k+1} \Delta_{\hat{g}}\left(\eta^{2}\right) d v_{\hat{g}} \\
& \quad-\frac{4 k}{(k+1)^{2}} \int_{M} w_{\alpha}^{k+1}\left|\nabla_{\hat{g}} \eta\right|^{2} d v_{\hat{g}}-\frac{k-1}{(k+1)^{2}} \int_{\partial M} w_{\alpha}^{k+1} \frac{\partial_{\hat{g}}\left(\eta^{2}\right)}{\partial \nu} d s_{\hat{g}}
\end{aligned}
$$


We derive from last two inequalities that

$$
\begin{aligned}
\int_{M}\left|\nabla_{\hat{g}}\left(w_{\alpha}^{k+1 / 2} \eta\right)\right|^{2} d v_{\hat{g}} & \\
\leq- & \frac{k-1}{4 k} \int_{M} w_{\alpha}^{k+1} \Delta_{\hat{g}}\left(\eta^{2}\right) d v_{\hat{g}}+\int_{M} w_{\alpha}^{k+1}\left|\nabla_{\hat{g}} \eta\right|^{2} d v_{\hat{g}} \\
& \quad+\frac{k-1}{4 k} \int_{\partial M} w_{\alpha}^{k+1} \frac{\partial_{\hat{g}}\left(\eta^{2}\right)}{\partial \nu} d s_{\hat{g}}+\frac{\xi_{\alpha}(k+1)^{2}}{4 k} \int_{M} w_{\alpha}^{p-1+k} \eta^{2} d v_{\hat{g}}
\end{aligned}
$$

For all $\epsilon>0$, it follows from Lemma 2.4 that there exists $\delta_{0}=\delta_{0}(\epsilon)>0$ such that

$$
\int_{M \backslash B_{\mu_{\alpha} / \delta_{0}}\left(x_{\alpha}\right)} w_{\alpha}^{p} d v_{\hat{g}}=\int_{M \backslash B_{\mu_{\alpha} / \delta_{0}}\left(x_{\alpha}\right)} u_{\alpha}^{p} d v_{g}<\epsilon .
$$

Then by the Moser iteration process (e.g. see the proof of Lemma $2.3 \mathrm{in}$ [LZ]), we know for $\epsilon_{0}$ small but independent of $\alpha$, there exists some $\delta_{0}=$ $\delta_{0}\left(\epsilon_{0}\right)>0$ and $s_{0}=s_{0}\left(\epsilon_{0}\right)>p$,

$$
\int_{M \backslash B_{\mu_{\alpha} / \delta_{0}}\left(x_{\alpha}\right)} w_{\alpha}^{s_{0}} d v_{\hat{g}} \leq C .
$$

Without loss of generality, we assume that $\delta_{0}>0$ is small enough so that $B_{\mu_{\alpha} / \delta_{0}}\left(x_{\alpha}\right) \subset B_{4 \mu_{\alpha} / \delta_{0}}\left(P_{\alpha}\right)$. Set, $\delta=\delta_{0} / 8$,

Clearly,

$$
R_{i}=\mu_{\alpha}\left(2-\frac{1}{2^{i-1}}\right) / \delta, \quad i=1,2,3, \ldots
$$

$$
B_{\mu_{\alpha} / \delta_{0}}\left(x_{\alpha}\right) \subset B_{R_{i}}\left(P_{\alpha}\right), \quad \forall i .
$$

Note that for $\mu_{\alpha} / \delta<|y| \leq 2 \mu_{\alpha} / \delta$,

$$
\mu_{\alpha}^{(2-n) / 2} / C \leq \varphi_{\alpha}(y) \leq C \mu_{\alpha}^{(2-n) / 2}, \quad C^{-1} \mu_{\alpha}^{-2} g \leq \hat{g} \leq C \mu_{\alpha}^{-2} g .
$$

We can choose some smooth cutoff function $\eta_{i}$ satisfying

$$
\left\{\begin{array}{l}
\eta_{i}(y)=1,|y|>R_{i+1} ; \quad \eta_{i}(y)=0,|y|<R_{i} ; \\
\left|\nabla_{\hat{g}} \eta_{i}\right| \leq C 2^{i}, \quad\left|\nabla_{\hat{g}}^{2} \eta_{i}\right| \leq C 4^{i} .
\end{array}\right.
$$

In the following we use notation $B_{R_{i}}=B_{R_{i}}\left(P_{\alpha}\right)$. Take $\eta=\eta_{i}$ in (2.29), we have,

$$
\begin{aligned}
\int_{M}\left|\nabla_{\hat{g}}\left(w_{\alpha}^{(k+1) / 2} \eta_{i}\right)\right|^{2} d v_{\hat{g}} & \\
\leq & 4^{i} C \int_{M \backslash B_{R_{i}}} w_{\alpha}^{k+1} d v_{\hat{g}}+2^{i} C \int_{\partial M \backslash B_{R_{i}}} w_{\alpha}^{k+1} d s_{\hat{g}} \\
& +\frac{(k+1)^{2} C}{k} \int_{M \backslash B_{R_{i}}} w_{\alpha}^{p-1+k} d v_{\hat{g}}
\end{aligned}
$$


Vol. 8, 1998 SHARP SOBOLEV INEQUALITIES INVOLVING BOUNDARY TERMS 73

It follows from the Sobolev embedding theorems (see Appendix A in [LZ]) that

$$
\begin{aligned}
& \left\{\int_{M \backslash B_{R_{i}}}\left(w_{\alpha}^{(k+1) / 2} \eta_{i}\right)^{p} d v_{\hat{g}}\right\}^{2 / p} \leq C \int_{M \backslash B_{R_{i}}}\left|\nabla_{\hat{g}}\left(w_{\alpha}^{(k+1) / 2} \eta_{i}\right)\right|^{2} d v_{\hat{g}} \\
& \left\{\int_{\partial M \backslash B_{R_{i}}}\left(w_{\alpha}^{(k+1) / 2} \eta_{i}\right)^{q} d s_{\hat{g}}\right\}^{2 / q} \leq C \int_{M \backslash B_{R_{i}}}\left|\nabla_{\hat{g}}\left(w_{\alpha}^{(k+1) / 2} \eta_{i}\right)\right|^{2} d v_{\hat{g}}
\end{aligned}
$$

Using (2.34) and (2.35), we can derive from (2.33) that

$$
\begin{gathered}
\left\{\int_{M \backslash B_{R_{i}}}\left(w_{\alpha}^{(k+1) / 2} \eta_{i}\right)^{p} d v_{\hat{g}}\right\}^{2 / p}+\left\{\int_{\partial M \backslash B_{R_{i}}}\left(w_{\alpha}^{(k+1) / 2} \eta_{i}\right)^{q} d s_{\hat{g}}\right\}^{2 / q} \\
\leq 4^{i} C \int_{M \backslash B_{R_{i}}} w_{\alpha}^{k+1} d v_{\hat{g}}+2^{i} C \int_{\partial M \backslash B_{R_{i}}} w_{\alpha}^{k+1} d s_{\hat{g}} \\
+\frac{(k+1)^{2} C}{k} \int_{M \backslash B_{R_{i}}} w_{\alpha}^{p-1+k} d v_{\hat{g}} .
\end{gathered}
$$

Set $r_{0}=s_{0} /(p-2)$, it follows from (2.30) and the Hölder's inequality that

$$
\int_{M \backslash B_{R_{i}}} w_{\alpha}^{p-1+k} d v_{\hat{g}} \leq C\left(\int_{M \backslash B_{R_{i}}} w_{\alpha}^{(k+1) r_{0} /\left(r_{0}-1\right)} d v_{\hat{g}}\right)^{\left(r_{0}-1\right) / r_{0}} .
$$

It follows from (2.36) and (2.37) that

$$
\begin{gathered}
\left\{\int_{M \backslash B_{R_{i+1}}} w_{\alpha}^{(k+1) p / 2} d v_{\hat{g}}\right\}^{2 / p}+\left\{\int_{\partial M \backslash B_{R_{i+1}}} w_{\alpha}^{(k+1) q / 2} d s_{\hat{g}}\right\}^{2 / q} \\
\leq 4^{i} C \int_{M \backslash B_{R_{i}}} w_{\alpha}^{k+1} d v_{\hat{g}}+2^{i} C \int_{\partial M \backslash B_{R_{i}}} w_{\alpha}^{k+1} d s_{\hat{g}} \\
+\frac{(k+1)^{2} C}{k}\left(\int_{M \backslash B_{R_{i}}} w_{\alpha}^{(k+1) r_{0} /\left(r_{0}-1\right)} d v_{\hat{g}}\right)^{\left(r_{0}-1\right) / r_{0}} .
\end{gathered}
$$

Set $\beta=\min \left\{p\left(r_{0}-1\right) /\left(2 r_{0}\right), q / 2\right\}$, it is easy to see from $s_{0}>p$ that $\beta>1$. It follows from Hölder's inequality that

$$
\begin{aligned}
& \left\{\int_{M \backslash B_{R_{i+1}}} w_{\alpha}^{\frac{(k+1) \beta r_{0}}{r_{0}-1}} d v_{\hat{g}}\right\}^{\frac{r_{0}-1}{\beta r_{0}}} \leq C\left\{\int_{M \backslash B_{R_{i+1}}} w_{\alpha}^{\frac{(k+1) p}{2}} d v_{\hat{g}}\right\}^{\frac{2}{p}}, \\
& \left\{\int_{\partial M \backslash B_{R_{i+1}}} w_{\alpha}^{(k+1) \beta} d s_{\hat{g}}\right\}^{1 / \beta} \leq C\left\{\int_{\partial M \backslash B_{R_{i+1}}} w_{\alpha}^{(k+1) q / 2} d s_{\hat{g}}\right\}^{2 / q},
\end{aligned}
$$


and

$$
\int_{M \backslash B_{R_{i}}} w_{\alpha}^{k+1} d v_{\hat{g}} \leq C\left\{\int_{M \backslash B_{R_{i}}} w_{\alpha}^{(k+1) r_{0} /\left(r_{0}-1\right)} d v_{\hat{g}}\right\}^{\left(r_{0}-1\right) / r_{0}} .
$$

It follows from (2.38), (2.39), (2.40), and (2.41) that

$$
\begin{aligned}
& \left\{\int_{M \backslash B_{R_{i+1}}} w_{\alpha}^{(k+1) \beta r_{0} /\left(r_{0}-1\right)} d v_{\hat{g}}\right\}^{\left(r_{0}-1\right) /\left(\beta r_{0}\right)}+\left\{\int_{\partial M \backslash B_{R_{i+1}}} w_{\alpha}^{(k+1) \beta} d s_{\hat{g}}\right\}^{1 / \beta} \\
& \left.\leq C\left(4^{i}+\frac{(k+1)^{2}}{k}\right)\left\{\int_{M \backslash B_{R_{i}}} w_{\alpha}^{\frac{(k+1) r_{0}}{r_{0}-1}} d v_{\hat{g}}\right)^{\frac{r_{0}-1}{r_{0}}}+\int_{\partial M \backslash B_{R_{i}}} w_{\alpha}^{k+1} d s_{\hat{g}}\right\} .
\end{aligned}
$$

Taking $k+1=2 \beta^{i}$ for $i \geq 1$ in the above, setting $p_{i}=2 \beta^{i} r_{0} /\left(r_{0}-1\right)$, $q_{i}=2 \beta^{i}$, we have

$$
\begin{aligned}
& \left\|w_{\alpha}\right\|_{p_{i+1}, M \backslash B_{R_{i+1}}}^{q_{i}}+\left\|w_{\alpha}\right\|_{q_{i+1}, \partial M \backslash B_{R_{i+1}}}^{q_{i}} \\
& \quad \leq C\left(4^{i}+\beta^{i}\right)\left(\left\|w_{\alpha}\right\|_{p_{i}, M \backslash B_{R_{i}}}^{q_{i}}+\left\|w_{\alpha}\right\|_{q_{i}, \partial M \backslash B_{R_{i}}}^{q_{i}}\right) .
\end{aligned}
$$

Since $\beta>1$, we have $a^{\beta}+b^{\beta} \leq(a+b)^{\beta}$ for all $a, b \geq 0$. It follows that

$$
\begin{aligned}
& \left(\left\|w_{\alpha}\right\|_{p_{i+1}, M \backslash B_{R_{i+1}}}^{q_{i+1}}+\left\|w_{\alpha}\right\|_{q_{i+1}, \partial M \backslash B_{R_{i+1}}}^{q_{i+1}}\right)^{1 / q_{i+1}} \\
& \quad \leq\left(\left\|w_{\alpha}\right\|_{p_{i+1}, M \backslash B_{R_{i+1}}}^{q_{i}}+\left\|w_{\alpha}\right\|_{q_{i+1}, \partial M \backslash B_{R_{i+1}}}^{q_{i}}\right)^{1 / q_{i}} \\
& \quad \leq\left(C 4^{i}+C \beta^{i}\right)^{1 / q_{i}}\left(\left\|w_{\alpha}\right\|_{p_{i}, M \backslash B_{R_{i}}}^{q_{i}}+\left\|w_{\alpha}\right\|_{q_{i}, \partial M \backslash B_{R_{i}}}^{q_{i}}\right)^{1 / q_{i}} .
\end{aligned}
$$

It is easy to see that

$$
\prod_{i=1}^{\infty}\left(C 4^{i}+C \beta^{i}\right)^{1 /\left(2 \beta^{i}\right)} \leq C<\infty .
$$

It follows that

$$
\left\|w_{\alpha}\right\|_{p_{i+1}, M \backslash B_{R_{i+1}}} \leq C\left(\left\|w_{\alpha}\right\|_{p_{1}, M \backslash B_{R_{1}}}^{2 \beta}+\left\|w_{\alpha}\right\|_{q_{1}, \partial M \backslash B_{R_{1}}}^{2 \beta}\right)^{1 /(2 \beta)} \leq C .
$$

Send $i$ to $\infty$, we have

$$
\left\|w_{\alpha}\right\|_{L^{\infty}\left(M \backslash B_{2 \mu_{\alpha} / \delta_{0}}\right)} \leq C\left(\delta_{0}\right) .
$$

It is easy to see that inside $B_{2 \mu_{\alpha} /\left(\delta_{0}\right)}\left(P_{\alpha}\right),|y| \leq C \mu_{\alpha}$. Therefore, it follows from the definition of $\varphi_{\alpha}$ and $(2.21)$ that $\varphi_{\alpha}(y) \geq C^{-1} \mu_{\alpha}^{-(n-2) / 2}$, $\forall y \in B_{2 \mu_{\alpha} /\left(\delta_{0}\right)}\left(P_{\alpha}\right)$. It follows that

$$
w_{\alpha}=u_{\alpha} / \varphi_{\alpha} \leq C \mu_{\alpha}^{(n-2) / 2} u_{\alpha}=C u_{\alpha} / u_{\alpha}\left(x_{\alpha}\right) \leq C, \quad \forall y \in B_{2 \mu_{\alpha} /\left(\delta_{0}\right)}\left(P_{\alpha}\right) .
$$

Proposition 2.1 follows from (2.43) and (2.44). 


\section{Balance Checking via Pohozaev Identity}

Let $Q_{\alpha} \in \partial M$ be the closest point on $\partial M$ to $x_{\alpha}$. By choosing an appropriate coordinate system centered at $Q_{\alpha}$, we can assume without loss of generality that $Q_{\alpha}=0, g_{i j}(0)=\delta_{i j}, B_{1}^{+}(0) \subset M,\left\{\left(x^{\prime}, 0\right):\left|x^{\prime}\right|<1\right\} \subset \partial M$.

Let $R_{\alpha}=1 /\left(\alpha \mu_{\alpha}\right), h_{\alpha}=g_{i j}\left(\mu_{\alpha} x\right) d x^{i} d x^{j}$ in $B_{10 R_{\alpha}}^{+}(0)$, and

$$
\bar{v}_{\alpha}(x)=\mu_{\alpha}^{(n-2) / 2} u_{\alpha}\left(\mu_{\alpha} x\right) \text { for } x \in B_{10 R_{\alpha}}^{+}(0) .
$$

It follows from (2.5) and (2.2) that $R_{\alpha} \rightarrow \infty$ as $\alpha \rightarrow \infty$, and $\bar{v}_{\alpha}$ satisfies

$$
\begin{cases}-\Delta_{h_{\alpha}} \bar{v}_{\alpha}=\xi_{\alpha} \bar{v}_{\alpha}^{p-1} & \text { in } B_{10 R_{\alpha}}^{+}(0) \\ \frac{\partial_{h_{\alpha}} \bar{v}_{\alpha}}{\partial \nu}=-\alpha \mu_{\alpha} \bar{v}_{\alpha} & \text { on }\left\{\left(x^{\prime}, 0\right):\left|x^{\prime}\right|<10 R_{\alpha}\right\} \\ 0<\bar{v}_{\alpha} \leq \mu_{\alpha}^{(n-2) / 2} u_{\alpha}(0) . & \end{cases}
$$

Clearly,

$$
\left|h_{\alpha}^{i j}(x)-\delta^{i j}\right| \leq C\left|\mu_{\alpha} x\right|, \quad\left|\Gamma_{i j}^{k}(x)\right| \leq C \mu_{\alpha} \quad \text { in } B_{10 R_{\alpha}}^{+}(0),
$$

where $\Gamma_{i j}^{k}$ is the Christoffel symbol of $h_{\alpha}$.

As in the proof of Lemma 2.2, $\left|x_{\alpha}-Q_{\alpha}\right| / \mu_{\alpha} \rightarrow 0$ as $\alpha \rightarrow \infty$. Therefore $\mu_{\alpha}^{(n-2) / 2} u_{\alpha}(0) \rightarrow 1$. As explained in section 2 ,

$$
\lim _{\alpha \rightarrow \infty}\left\|\bar{v}_{\alpha}-v\right\|_{C^{2}\left(\overline{B_{R}^{+}(0)}\right)}=0, \quad \forall R>1,
$$

where $v$ is the function defined in $\mathbb{R}_{+}^{n}$, given in (2.13). It is not difficult to see from Proposition 2.1 that

$$
\bar{v}_{\alpha}(x) \leq \frac{C}{1+|x|^{n-2}} \quad \text { for } \quad x \in \overline{B_{10 R_{\alpha}}^{+}}(0) .
$$

We need some further estimates on $\bar{v}_{\alpha}$.

Lemma 3.1. For all $\alpha \geq 1, x \in B_{R_{\alpha}}^{+}(0)$, we have

$$
\left|\nabla \bar{v}_{\alpha}(x)\right| \leq \frac{C}{1+|x|^{n-1}}, \quad\left|\nabla^{2} \bar{v}_{\alpha}(x)\right| \leq \frac{C}{1+|x|^{n}},
$$

where $\left|\nabla^{2} \bar{v}_{\alpha}\right|=\sum_{i, j=1}^{n}\left|\partial^{2} \bar{v}_{\alpha} / \partial x^{i} \partial x^{j}\right|$, and $C$ is some constant independent of $\alpha$ and $x$.

Proof. It follows from (3.3) that

$$
\left|\nabla \bar{v}_{\alpha}(x)\right|<C, \quad\left|\nabla^{2} \bar{v}_{\alpha}(x)\right| \leq C, \text { in } B_{1}^{+}(0) .
$$

So we only need to show Lemma 3.1 for $|x|>1$. For all $x_{0} \in B_{R_{\alpha}}^{+}(0) \backslash B_{1}^{+}(0)$, set $R=\left|x_{0}\right|, \tilde{u}(x)=R^{n-2} \bar{v}_{\alpha}(R x)$ and $\tilde{g}_{i j}(x)=\left(h_{\alpha}\right)_{i j}(R x)$. It follows from 
(3.1) that

$$
\begin{cases}-\Delta_{\tilde{g}} \tilde{u}=R^{n} \xi_{\alpha} \bar{v}_{\alpha}(R x)^{p-1} & \text { in } B_{5}^{+}(0) \backslash B_{1 / 5}^{+}(0) \\ \frac{\partial_{\tilde{g}} \tilde{u}}{\partial \nu}=-R^{n-1} \alpha \mu_{\alpha} \bar{v}_{\alpha}(R x) & \text { on }\left\{\left(x^{\prime}, 0\right): 1 / 5<\left|x^{\prime}\right|<5\right\} .\end{cases}
$$

From (3.4) we can derive that: In $B_{5}^{+}(0) \backslash B_{1 / 5}^{+}(0)$

$$
|\tilde{u}(x)| \leq C, \quad\left|R^{n} \xi_{\alpha} \bar{v}_{\alpha}(R x)^{p-1}\right| \leq C R^{-2} \leq C .
$$

On $\left\{\left(x^{\prime}, 0\right): 1 / 5<\left|x^{\prime}\right|<5\right\}$

$$
\left.\left|\frac{\partial_{\tilde{g}} \tilde{u}}{\partial \nu}\right|=\mid-R^{n-1} \alpha \mu_{\alpha} \bar{v}_{\alpha}(R x)\right) \mid \leq C \alpha \mu_{\alpha} R \leq C .
$$

It follows from standard elliptic estimates that for some $0<\beta<1$,

$$
\|\tilde{u}\|_{C^{\beta}\left(\overline{B_{4}^{+} \backslash B_{1 / 4}^{+}}\right)} \leq C \text {. }
$$

Rewriting the equations of $\tilde{u}$ as

$$
\begin{cases}-\Delta_{\tilde{g}} \tilde{u}=R^{-2} \xi_{\alpha} \tilde{u}^{p-1} & \text { in } B_{5}^{+}(0) \backslash B_{1 / 5}^{+}(0) \\ \frac{\partial_{\tilde{g}} \tilde{u}}{\partial \nu}=-\alpha \mu_{\alpha} R \tilde{u}, & \text { on }\left\{\left(x^{\prime}, 0\right): 1 / 5<\left|x^{\prime}\right|<5\right\},\end{cases}
$$

and noticing (see (3.5))

$$
\left\|R^{-2} \xi_{\alpha} \tilde{u}^{p-1}\right\|_{C^{\beta}\left(\overline{B_{4}^{+} \backslash B_{1 / 4}^{+}}\right)} \leq C, \quad\left\|\alpha \mu_{\alpha} R \tilde{u}\right\|_{C^{\beta}\left(\overline{B_{4}^{+} \backslash B_{1 / 4}^{+}}\right)} \leq C,
$$

we have, by standard elliptic estimates, that

$$
\|\tilde{u}\|_{C^{2, \beta}\left(\overline{B_{3}^{+} \backslash B_{1 / 3}^{+}}\right)} \leq C .
$$

Therefore

$$
\left|\nabla \bar{v}_{\alpha}\left(x_{0}\right)\right|=R^{1-n}\left|\nabla_{\tilde{g}} \tilde{u}\left(x_{0} / R\right)\right| \leq C\left|x_{0}\right|^{1-n} \leq C /\left(1+\left|x_{0}\right|^{n-1}\right),
$$

which gives us the gradient estimate.

Also the second derivatives can be estimated as

$$
\left|\nabla^{2} \bar{v}_{\alpha}\left(x_{0}\right)\right|=R^{-n}\left|\nabla^{2} \tilde{u}\left(x_{0} / R\right)\right| \leq C\left|x_{0}\right|^{-n} \leq C /\left(1+\left|x_{0}\right|^{n}\right) .
$$

For $n=3$, we need to obtain an appropriate lower bound of $\bar{v}_{\alpha}$ in order to reach a contradiction from the balance checking by using the Pohozaev type identity.

Lemma 3.2. For $n=3$, as $\alpha$ large enough,

$$
\bar{v}_{\alpha}(x) \geq \frac{1}{C(1+|x|)}, \quad \forall x \in \overline{B_{R_{\alpha}^{1 / 4}}^{+}(0)},
$$

where $C>0$ is some constant independent of $\alpha$. 
Proof. In view of (3.3), we only need to prove the above estimate for $|x|>20$. In the following, $\alpha$ is always assumed to be suitably large. Let $\bar{x}=(0, \ldots, 0,1)$ and

$$
G_{\alpha}(x)=\frac{1}{|x-\bar{x}|}-\frac{1}{R_{\alpha}^{1 / 2}|x-\bar{x}|^{1 / 2}} \quad \text { in } \quad B_{R_{\alpha}^{1 / 3}}(\bar{x}) \backslash B_{2}(\bar{x}) .
$$

It is easy to see that

$$
\frac{1}{2|x-\bar{x}|} \leq G_{\alpha}(x) \leq \frac{2}{|x-\bar{x}|} \quad \text { in } \quad B_{R_{\alpha}^{1 / 3}}(\bar{x}) \backslash B_{2}(\bar{x}) .
$$

Using (3.2), it is easy to see that in $B_{R_{\alpha}^{1 / 3}}(\bar{x}) \backslash B_{2}(\bar{x})$

$$
\Delta_{h_{\alpha}}\left(-\frac{1}{R_{\alpha}^{1 / 2}|x-\bar{x}|^{1 / 2}}\right) \geq \frac{1}{C R_{\alpha}^{1 / 2}|x-\bar{x}|^{5 / 2}}, \quad\left|\Delta_{h_{\alpha}}\left(\frac{1}{|x-\bar{x}|}\right)\right| \leq \frac{C \mu_{\alpha}}{|x-\bar{x}|^{2}} .
$$

It follows that $\Delta_{h_{\alpha}} G_{\alpha} \geq 0$.

Also, it follows from (3.2) that for all $x=\left(x^{\prime}, 0\right), 1<\left|x^{\prime}\right|<R_{\alpha}^{1 / 3}$,

$$
\begin{gathered}
\frac{\partial_{h_{\alpha}}}{\partial \nu}\left(\frac{1}{|x-\bar{x}|}\right) \leq-\frac{1}{C|x-\bar{x}|^{3}}, \\
\left|\frac{\partial_{h_{\alpha}}}{\partial \nu}\left(\frac{1}{R_{\alpha}^{1 / 2}|x-\bar{x}|^{1 / 2}}\right)\right| \leq \frac{C}{R_{\alpha}^{1 / 4}|x-\bar{x}|^{3}} .
\end{gathered}
$$

Therefore, using (3.4) also, we have

$$
\begin{gathered}
-\alpha \mu_{\alpha} \bar{v}_{\alpha}-\frac{\partial_{h_{\alpha}}}{\partial \nu}\left(G_{\alpha}\right) \geq-\frac{C}{R_{\alpha}(1+|x|)}+\frac{1}{C|x-\bar{x}|^{3}} \geq 0, \\
\forall x=\left(x^{\prime}, 0\right), 1<\left|x^{\prime}\right|<R_{\alpha}^{1 / 3} .
\end{gathered}
$$

We will use the maximum principle on $A=\left\{x \in \mathbb{R}_{+}^{n}: 10<|x-\bar{x}|<R_{\alpha}^{1 / 3}\right\}$. Let $\Sigma_{1}=\partial A \cap\left\{x_{n}=0\right\}, \Sigma_{2}=\partial A \cap\{|x-\bar{x}|=10\}$, and $\Sigma_{3}=\partial A \cap\{|x-\bar{x}|=$ $\left.R_{\alpha}^{1 / 3}\right\}$. Choose $0<\tau<1$ small enough (independent of $\alpha$ ) such that $\tau G_{\alpha} \leq \bar{v}_{\alpha}$ on $\Sigma_{2}$. Define $H_{\alpha}=\tau G_{\alpha}-\max _{\Sigma_{3}}\left(\tau G_{\alpha}\right)$, then

$$
\begin{cases}\Delta_{h_{\alpha}}\left(\bar{v}_{\alpha}-H_{\alpha}\right) \leq 0, & \text { in } A \\ \bar{v}_{\alpha}-H_{\alpha} \geq 0, & \text { on } \Sigma_{2} \cup \Sigma_{3} \\ \frac{\partial_{h_{\alpha}}\left(\bar{v}_{\alpha}-H_{\alpha}\right)}{\partial \nu}>0, & \text { on } \Sigma_{1}\end{cases}
$$

It follows from the maximum principle that

$$
\bar{v}_{\alpha} \geq H_{\alpha} \quad \text { in } A .
$$

Consequently,

$$
\bar{v}_{\alpha}(x) \geq H_{\alpha}(x) \geq \frac{C \tau}{|x-\bar{x}|}-\frac{C \tau}{R_{\alpha}^{1 / 3}} \geq \frac{C \tau}{2|x-\bar{x}|}, \quad \forall x \in B_{R_{\alpha}^{1 / 4}}^{+}(0) \backslash B_{10}^{+}(\bar{x}) .
$$


Lemma 3.2 is established.

For convenience, throughout the rest of this section we set $\Gamma_{1}=$ $\partial B_{R_{\alpha}}^{+}(0) \cap\left\{\left(x^{\prime}, 0\right): x^{\prime} \in \mathbb{R}^{n-1}\right\}, \Gamma_{2}=\partial B_{R_{\alpha}}^{+}(0) \cap\left\{\left(x^{\prime}, x_{n}\right): x_{n}>0\right\}$. We always use $d V$ for the volume element of the standard Euclidean metric, $d S$ for the surface element of the standard Euclidean metric, $\nu$ for the unit outer normal vector of the corresponding surface with respect to the specified metrics, and "." for the inner product under the standard Euclidean metric.

As in [LZ], we have the following identity,

$$
\int_{B_{R_{\alpha}}^{+}} \Delta \bar{v}_{\alpha}\left(\nabla \bar{v}_{\alpha} \cdot x\right) d V+\frac{n-2}{2} \int_{B_{R_{\alpha}}^{+}} \bar{v}_{\alpha} \Delta \bar{v}_{\alpha} d V=J\left(R_{\alpha}, \bar{v}_{\alpha}\right)+I\left(R_{\alpha}, \bar{v}_{\alpha}\right),
$$

where

$$
\begin{aligned}
& J\left(R_{\alpha}, \bar{v}_{\alpha}\right)=\frac{1}{2} \int_{\Gamma_{2}}\left\{\left|\frac{\partial \bar{v}_{\alpha}}{\partial \nu}\right|^{2}|x|-\left|\partial_{\tan } \bar{v}_{\alpha}\right|^{2}|x|+(n-2) \frac{\partial \bar{v}_{\alpha}}{\partial \nu} \bar{v}_{\alpha}\right\} d S \\
& I\left(R_{\alpha}, \bar{v}_{\alpha}\right)=\frac{1}{2} \int_{\Gamma_{1}}\left\{2\left(\sum_{i=1}^{n-1} x_{i} \frac{\partial \bar{v}_{\alpha}}{\partial x_{i}}\right) \frac{\partial \bar{v}_{\alpha}}{\partial \nu}+(n-2) \frac{\partial \bar{v}_{\alpha}}{\partial \nu} \bar{v}_{\alpha}\right\} d S .
\end{aligned}
$$

Replacing $\Delta \bar{v}_{\alpha}$ in (3.7) by

$$
\Delta \bar{v}_{\alpha}=\Delta_{h_{\alpha}} \bar{v}_{\alpha}-\left(h_{\alpha}^{i j}-\delta^{i j}\right) \partial_{i j} \bar{v}_{\alpha}+h_{\alpha}^{i j} \Gamma_{i j}^{k} \partial_{k} \bar{v}_{\alpha}
$$

we have

$$
\begin{aligned}
& -\int_{B_{R_{\alpha}}^{+}}\left(x^{i} \partial_{i} \bar{v}_{\alpha}\right) \Delta_{h_{\alpha}} \bar{v}_{\alpha} d V-\frac{n-2}{2} \int_{B_{R_{\alpha}}^{+}} \bar{v}_{\alpha} \Delta_{h_{\alpha}} \bar{v}_{\alpha} d V \\
& +\int_{B_{R_{\alpha}}^{+}}\left(x^{k} \partial_{k} \bar{v}_{\alpha}\right)\left(h_{\alpha}^{i j}-\delta^{i j}\right) \partial_{i j} \bar{v}_{\alpha} d V-\int_{B_{R_{\alpha}}^{+}}\left(x^{l} \partial_{l} \bar{v}_{\alpha}\right)\left(h_{\alpha}^{i j} \Gamma_{i j}^{k} \partial_{k} \bar{v}_{\alpha}\right) d V \\
& \quad+\frac{n-2}{2} \int_{B_{R_{\alpha}}^{+}} \bar{v}_{\alpha}\left(h_{\alpha}^{i j}-\delta^{i j}\right) \partial_{i j} \bar{v}_{\alpha} d V-\frac{n-2}{2} \int_{B_{R_{\alpha}}^{+}} \bar{v}_{\alpha}\left(h_{\alpha}^{i j} \Gamma_{i j}^{k}\right) \partial_{k} \bar{v}_{\alpha} d V \\
& \quad=-J\left(R_{\alpha}, \bar{v}_{\alpha}\right)-I\left(R_{\alpha}, \bar{v}_{\alpha}\right) .
\end{aligned}
$$

So far, we have not used the equation of $\bar{v}_{\alpha}$ yet. Now we use equation (3.1) satisfied by $\bar{v}_{\alpha}$ and obtain

$$
A\left(h_{\alpha}, \bar{v}_{\alpha}\right)=-J\left(R_{\alpha}, \bar{v}_{\alpha}\right)-I\left(R_{\alpha}, \bar{v}_{\alpha}\right),
$$


where

$$
\begin{aligned}
& A\left(h_{\alpha}, \bar{v}_{\alpha}\right)=\frac{\xi_{\alpha}}{p} \int_{\Gamma_{2}} \bar{v}_{\alpha}^{p}|x| d S \\
& \quad+\int_{B_{R_{\alpha}}^{+}}\left(x^{k} \partial_{k} \bar{v}_{\alpha}\right)\left(h_{\alpha}^{i j}-\delta^{i j}\right) \partial_{i j} \bar{v}_{\alpha} d V-\int_{B_{R_{\alpha}}^{+}}\left(x^{l} \partial_{l} \bar{v}_{\alpha}\right)\left(h_{\alpha}^{i j} \Gamma_{i j}^{k} \partial_{k} \bar{v}_{\alpha}\right) d V \\
& \quad+\frac{n-2}{2} \int_{B_{R_{\alpha}}^{+}} \bar{v}_{\alpha}\left(h_{\alpha}^{i j}-\delta^{i j}\right) \partial_{i j} \bar{v}_{\alpha} d V-\frac{n-2}{2} \int_{B_{R_{\alpha}}^{+}} \bar{v}_{\alpha}\left(h_{\alpha}^{i j} \Gamma_{i j}^{k}\right) \partial_{k} \bar{v}_{\alpha} d V .
\end{aligned}
$$

Consequently, by using (3.2), we have

$$
\begin{aligned}
& A\left(h_{\alpha}, \bar{v}_{\alpha}\right)=O\left(\int_{\Gamma_{2}} \bar{v}_{\alpha}^{p}|x| d S\right) \\
& +O\left(\int_{B_{R_{\alpha}}^{+}} \mu_{\alpha}|x|^{2}\left|\nabla \bar{v}_{\alpha}\right|\left|\nabla^{2} \bar{v}_{\alpha}\right| d V\right)+O\left(\int_{B_{R_{\alpha}}^{+}} \mu_{\alpha}|x|\left|\nabla \bar{v}_{\alpha}\right|^{2} d V\right) \\
& \quad+O\left(\int_{B_{R_{\alpha}}^{+}} \mu_{\alpha}|x| \bar{v}_{\alpha}\left|\nabla^{2} \bar{v}_{\alpha}\right| d V\right)+O\left(\int_{B_{R_{\alpha}}^{+}} \mu_{\alpha} \bar{v}_{\alpha}\left|\nabla \bar{v}_{\alpha}\right| d V\right)
\end{aligned}
$$

We simplify $I\left(R_{\alpha}, \bar{v}_{\alpha}\right)$ by using the boundary condition in (3.1). It is easy to see from (3.2) that

$$
\frac{\partial_{h_{\alpha}} \bar{v}_{\alpha}}{\partial \nu}=\frac{\partial \bar{v}}{\partial \nu}+O\left(\mu_{\alpha}\left|x^{\prime}\right|\left|\nabla \bar{v}_{\alpha}\right|\right) \text { on } \Gamma_{1} .
$$

It follows that

$$
\begin{aligned}
2 I\left(R_{\alpha}, \bar{v}_{\alpha}\right)=\int_{\Gamma_{1}} & \left\{2\left(\sum_{i=1}^{n-1} x_{i} \frac{\partial \bar{v}_{\alpha}}{\partial x_{i}}\right) \frac{\partial_{h_{\alpha}} \bar{v}_{\alpha}}{\partial \nu}+(n-2) \frac{\partial_{h_{\alpha}} \bar{v}_{\alpha}}{\partial \nu} \bar{v}_{\alpha}\right\} d S \\
+ & O\left(\int_{\Gamma_{1}}\left[\mu_{\alpha}\left|x^{\prime}\right|^{2}\left|\nabla \bar{v}_{\alpha}\right|^{2}+\mu_{\alpha}\left|x^{\prime}\right| \bar{v}_{\alpha}\left|\nabla \bar{v}_{\alpha}\right|\right] d S\right) .
\end{aligned}
$$

Using the boundary condition in (3.1), we have

$$
\begin{aligned}
\int_{\Gamma_{1}} & \left\{2\left(\sum_{i=1}^{n-1} x_{i} \frac{\partial \bar{v}_{\alpha}}{\partial x_{i}}\right) \frac{\partial_{h_{\alpha}} \bar{v}_{\alpha}}{\partial \nu}+(n-2) \frac{\partial_{h_{\alpha}} \bar{v}_{\alpha}}{\partial \nu} \bar{v}_{\alpha}\right\} d S \\
\quad= & \int_{\Gamma_{1}}\left\{-2 \alpha \mu_{\alpha} \bar{v}_{\alpha}\left(\sum_{i=1}^{n-1} x_{i} \frac{\partial \bar{v}_{\alpha}}{\partial x_{i}}\right)-(n-2) \alpha \mu_{\alpha} \bar{v}_{\alpha}^{2}\right\} d S \\
= & (n-1) \alpha \mu_{\alpha} \int_{\Gamma_{1}} \bar{v}_{\alpha}^{2} d S-\int_{\partial \Gamma_{1}} \alpha \mu_{\alpha} \bar{v}_{\alpha}^{2}|x| d S-(n-2) \alpha \mu_{\alpha} \int_{\Gamma_{1}} \bar{v}_{\alpha}^{2} d S \\
= & \alpha \mu_{\alpha} \int_{\Gamma_{1}} \bar{v}_{\alpha}^{2} d S-\int_{\partial \Gamma_{1}} \alpha \mu_{\alpha} \bar{v}_{\alpha}^{2}|x| d S .
\end{aligned}
$$


Thus

$$
\begin{aligned}
& I\left(R_{\alpha}, \bar{v}_{\alpha}\right)=\frac{\alpha \mu_{\alpha}}{2} \int_{\Gamma_{1}} \bar{v}_{\alpha}^{2} d S+O\left(\int_{\partial \Gamma_{1}} \alpha \mu_{\alpha} \bar{v}_{\alpha}^{2}|x| d S\right) \\
& \quad+O\left(\int_{\Gamma_{1}}\left[\mu_{\alpha}\left|x^{\prime}\right|^{2}\left|\nabla \bar{v}_{\alpha}\right|^{2}+\mu_{\alpha}\left|x^{\prime}\right| \bar{v}_{\alpha}\left|\nabla \bar{v}_{\alpha}\right|\right] d S\right)
\end{aligned}
$$

Clearly,

$$
J\left(R_{\alpha}, \bar{v}_{\alpha}\right)=O\left(\int_{\Gamma_{2}}\left(|x|\left|\nabla \bar{v}_{\alpha}\right|^{2}+\bar{v}_{\alpha}\left|\nabla \bar{v}_{\alpha}\right|\right) d S\right) .
$$

In view of all the above estimates, we can rewrite (3.10) as the following Pohozaev type identity

$$
\begin{aligned}
\alpha \mu_{\alpha} \int_{\Gamma_{1}} \bar{v}_{\alpha}^{2} d S=O\left(\int_{\Gamma_{2}} \bar{v}_{\alpha}^{p}|x| d S\right) \\
+O\left(\int_{B_{R_{\alpha}}^{+}} \mu_{\alpha}|x|^{2}\left|\nabla \bar{v}_{\alpha}\right|\left|\nabla^{2} \bar{v}_{\alpha}\right| d V\right)+O\left(\int_{B_{R_{\alpha}}^{+}} \mu_{\alpha}|x|\left|\nabla \bar{v}_{\alpha}\right|^{2} d V\right) \\
+O\left(\int_{B_{R_{\alpha}}^{+}} \mu_{\alpha}|x| \bar{v}_{\alpha}\left|\nabla^{2} \bar{v}_{\alpha}\right| d V\right)+O\left(\int_{B_{R_{\alpha}}^{+}} \mu_{\alpha} \bar{v}_{\alpha}\left|\nabla \bar{v}_{\alpha}\right| d V\right) \\
+O\left(\int_{\Gamma_{2}}\left(|x|\left|\nabla \bar{v}_{\alpha}\right|^{2}+\bar{v}_{\alpha}\left|\nabla \bar{v}_{\alpha}\right|\right) d S\right) \\
+O\left(\int_{\partial \Gamma_{1}} \alpha \mu_{\alpha} \bar{v}_{\alpha}^{2}|x| d S\right) \\
\quad+O\left(\int_{\Gamma_{1}}\left[\mu_{\alpha}\left|x^{\prime}\right|^{2}\left|\nabla \bar{v}_{\alpha}\right|^{2}+\mu_{\alpha}\left|x^{\prime}\right| \bar{v}_{\alpha}\left|\nabla \bar{v}_{\alpha}\right|\right] d S\right) .
\end{aligned}
$$

We will derive a contradiction from (3.16) by showing that the left hand side is much large than the right hand side.

Lemma 3.3. For $n \geq 3$, there exists some constant $C>0$ independent of $\alpha$, such that $\int_{\Gamma_{1}} \bar{v}_{\alpha}^{2} d S>1 / C$ for all $\alpha \geq 1$. Moreover for $n=3$, $\int_{\Gamma_{1}} \bar{v}_{\alpha}^{2} d S \geq\left(\log R_{\alpha}\right) / C$ for all $\alpha \geq 1$.

Proof. Clearly we only need to prove the lemma for large $\alpha$. It follows easily from (3.3) that

$$
\int_{\Gamma_{1}} \bar{v}_{\alpha}^{2} d S \geq 1 / C
$$

For $n=3$, it follows from Lemma 3.2 that

$$
\int_{\Gamma_{1}} \bar{v}_{\alpha}^{2} d S \geq \frac{1}{C} \int_{\partial \mathbb{R}_{+}^{3} \cap B_{R_{\alpha}^{1 / 4}}}\left(\frac{1}{1+|x|}\right)^{2} d S \geq\left(\log R_{\alpha}\right) / C
$$


LEMma 3.4. The following estimates holds:

$$
\begin{aligned}
& \int_{\partial \Gamma_{1}} \alpha \mu_{\alpha} \bar{v}_{\alpha}^{2}|x| d S \leq \alpha \mu_{\alpha} R_{\alpha}^{3-n}, \\
& \int_{\Gamma_{1}}\left(\mu_{\alpha}\left|x^{\prime}\right|^{2}\left|\nabla \bar{v}_{\alpha}\right|^{2}+\mu_{\alpha}\left|x^{\prime}\right| \bar{v}_{\alpha}\left|\nabla \bar{v}_{\alpha}\right|\right) d S \leq \begin{cases}C \mu_{\alpha} \log R_{\alpha}, & n=3, \\
C \mu_{\alpha}, & n \geq 4,\end{cases} \\
& \int_{\Gamma_{2}}\left(|x|\left|\nabla \bar{v}_{\alpha}\right|^{2}+\bar{v}_{\alpha}\left|\nabla \bar{v}_{\alpha}\right|\right) d S \leq C\left(\alpha \mu_{\alpha}\right)^{n-2}, \\
& \int_{\Gamma_{2}} \bar{v}_{\alpha}^{p}|x| d S \leq C\left(\alpha \mu_{\alpha}\right)^{n}, \\
& \int_{B_{R_{\alpha}}^{+}}\left(\mu_{\alpha}|x|^{2}\left|\nabla \bar{v}_{\alpha}\right|\left|\nabla^{2} \bar{v}_{\alpha}\right|+\mu_{\alpha}|x|\left|\nabla \bar{v}_{\alpha}\right|^{2}\right) d V \leq \begin{cases}C \mu_{\alpha} \log R_{\alpha}, & n=3, \\
C \mu_{\alpha}, & n \geq 4,\end{cases} \\
& \int_{B_{R_{\alpha}}^{+}}\left(\mu_{\alpha}|x| \bar{v}_{\alpha}\left|\nabla^{2} \bar{v}_{\alpha}\right|+\mu_{\alpha} \bar{v}_{\alpha}\left|\nabla \bar{v}_{\alpha}\right|\right) d V \leq \begin{cases}C \mu_{\alpha} \log R_{\alpha}, & n=3, \\
C \mu_{\alpha}, & n \geq 4 .\end{cases}
\end{aligned}
$$

Proof. It follows easily from (3.4), Lemma 3.1, and some elementary calculation.

Proof of the sufficient part of Theorem 0.1. We draw a contradiction from (3.16) using Lemma 3.3 and Lemma 3.4. Since the left hand side is clearly much larger than the right hand side in (3.16) as $\alpha$ tends to infinity.

\section{Proof of Theorem 0.2}

We now begin to prove Theorem 0.2 by a contradiction argument. Suppose Theorem 0.2 were false, we would have $\forall \alpha \geq 1$,

$$
\zeta_{\alpha}:=\inf _{H^{1}(M) \backslash\{0\}} \frac{\int_{M}\left|\nabla_{g} u\right|^{2} d v_{g}+\alpha \int_{M} u^{2} d v_{g}+\alpha \int_{\partial M} u^{2} d s_{g}}{\left(\int_{M}|u|^{p} d v_{g}\right)^{2 / p}}<\frac{1}{2^{2 / n} S_{1}} .
$$

As shown in section 1 , we know that there exists some nonnegative $u_{\alpha} \in H^{1}(M)$ such that

$$
\zeta_{\alpha}=\int_{M}\left|\nabla_{g} u\right|^{2} d v_{g}+\alpha \int_{M} u^{2} d v_{g}+\alpha \int_{\partial M} u^{2} d s_{g}, \quad \int_{M} u_{\alpha}^{p} d v_{g}=1 .
$$

Therefore, $u_{\alpha}$ satisfies

$$
\begin{cases}-\Delta_{g} u_{\alpha}=\zeta_{\alpha} u_{\alpha}^{p-1}-\alpha u_{\alpha}, & \text { in } M \\ \frac{\partial_{g} u_{\alpha}}{\partial \nu}=-\alpha u_{\alpha}, & \text { on } \partial M\end{cases}
$$


For any $\epsilon>0$, it follows from (4.1), (4.2) and Proposition 1.1 that there exists some $B(\epsilon)$ such that

$$
\begin{aligned}
1+\frac{\epsilon}{2^{2 / n} S_{1}}> & \left(2^{2 / n} S_{1}+\epsilon\right) \zeta_{\alpha} \\
= & \left(2^{2 / n} S_{1}+\epsilon\right)\left\|\nabla_{g} u_{\alpha}\right\|_{2, M}^{2} \\
& \quad+\alpha\left(2^{2 / n} S_{1}+\epsilon\right)\left\|u_{\alpha}\right\|_{2, \partial M}^{2}+\alpha\left(2^{2 / n} S_{1}+\epsilon\right)\left\|u_{\alpha}\right\|_{2, M}^{2} \\
\geq & \left(\int_{M}\left|u_{\alpha}\right|^{p}\right)^{2 / p}+\alpha\left(2^{2 / n} S_{1}+\epsilon\right)\left\|u_{\alpha}\right\|_{2, \partial M}^{2} \\
& \quad+\left[\alpha\left(2^{2 / n} S_{1}+\epsilon\right)-B(\epsilon)\right]\left\|u_{\alpha}\right\|_{2, M}^{2} \\
= & +\alpha\left(2^{2 / n} S_{1}+\epsilon\right)\left\|u_{\alpha}\right\|_{2, \partial M}^{2}+\left[\alpha\left(2^{2 / n} S_{1}+\epsilon\right)-B(\epsilon)\right]\left\|u_{\alpha}\right\|_{2, M}^{2} .
\end{aligned}
$$

Thus

$$
\begin{aligned}
1+\frac{\epsilon}{2^{2 / n} S_{1}} \geq & \left(2^{2 / n} S_{1}+\epsilon\right) \limsup _{\alpha \rightarrow \infty} \zeta_{\alpha} \\
\geq 1 & +\left(2^{2 / n} S_{1}+\epsilon\right) \limsup _{\alpha \rightarrow \infty} \alpha\left\|u_{\alpha}\right\|_{2, \partial M}^{2} \\
& +\left(2^{2 / n} S_{1}+\epsilon\right) \limsup _{\alpha \rightarrow \infty} \alpha\left\|u_{\alpha}\right\|_{2, M}^{2}, \\
1+\frac{\epsilon}{2^{2 / n} S_{1}} \geq & \left(2^{2 / n} S_{1}+\epsilon\right) \liminf _{\alpha \rightarrow \infty} \zeta_{\alpha} \\
\geq 1 & +\left(2^{2 / n} S_{1}+\epsilon\right) \liminf _{\alpha \rightarrow \infty} \alpha\left\|u_{\alpha}\right\|_{2, \partial M}^{2} \\
& +\left(2^{2 / n} S_{1}+\epsilon\right) \liminf _{\alpha \rightarrow \infty} \alpha\left\|u_{\alpha}\right\|_{2, M}^{2} .
\end{aligned}
$$

Send $\epsilon \rightarrow 0$, we have

$$
\begin{gathered}
\lim _{\alpha \rightarrow \infty} \zeta_{\alpha}=\frac{1}{2^{2 / n} S_{1}}, \\
\lim _{\alpha \rightarrow \infty} \alpha\left\|u_{\alpha}\right\|_{2, \partial M}^{2}=0, \quad \lim _{\alpha \rightarrow \infty} \alpha\left\|u_{\alpha}\right\|_{2, M}^{2}=0 .
\end{gathered}
$$

It follows from Cherrier [Che1] that $u_{\alpha}$ is smooth up to boundary. Let $x_{\alpha}$ be some maximum point of $u_{\alpha}$, we set $\mu_{\alpha}=u_{\alpha}^{-2 /(n-2)}\left(x_{\alpha}\right)$.

Now we prove the following asymptotic behavior about $\mu_{\alpha}$.

LeMma 4.1.

$$
\lim _{\alpha \rightarrow \infty} \alpha \mu_{\alpha}=0
$$

Proof. The proof is similar to that of Lemma 2.1. First we claim

$$
\liminf _{\alpha \rightarrow \infty}\left\|u_{\alpha}\right\|_{q, \partial M}>0 .
$$


If the claim were false, i.e., $\left\|u_{\alpha}\right\|_{q, \partial M} \rightarrow 0$ along a subsequence $\alpha \rightarrow \infty$, then, in view of (4.1) and (4.2), there would exist $\bar{u} \in H_{0}^{1}(M)$ such that $u_{\alpha}$ weakly converges to $\bar{u}$. It follows from Proposition 1.3 applied to $u_{\alpha}-\bar{u}$, the compact embedding of $H^{1}(M)$ to $L^{2}(M)$, the definition of $\zeta,(1.7)$ and (1.8), that for $\epsilon>0$

$$
\begin{aligned}
\zeta_{\alpha} & =\int_{M}\left|\nabla_{g} u_{\alpha}\right|^{2}+\alpha \int_{\partial M} u_{\alpha}^{2}+\alpha \int_{M} u_{\alpha}^{2} \\
& \geq\left(1 / S_{1}-\epsilon-\zeta_{\alpha}\right) \int_{M}\left|u_{\alpha}-\bar{u}\right|^{p}+\zeta_{\alpha}+\alpha \int_{\partial M} u_{\alpha}^{2}+\alpha \int_{M} u_{\alpha}^{2}+o(1),
\end{aligned}
$$

where $o(1) \rightarrow 0$ as $\alpha \rightarrow \infty$. Take $\epsilon$ small enough, we derive $\left\|u_{\alpha}-\bar{u}\right\|_{p, M} \rightarrow 0$, therefore $\| \bar{u}||_{p, M}=1$. But from (4.5) we know $\|\bar{u}\|_{2, M}=0$. Contradiction.

Similar to the proof of Proposition 2.1, we have

Proposition 4.1. There exists some constant $C$ such that for all $\alpha \geq 1$

$$
u_{\alpha} / \varphi_{\alpha} \leq C \text { for } x \in \bar{M},
$$

where $\varphi_{\alpha}$ was defined in (2.22).

Let $Q_{\alpha} \in \partial M$ be the closest point to $x_{\alpha}$. By choosing an appropriate coordinate system centered at $Q_{\alpha}$, we can assume without loss of generality that $Q_{\alpha}=0, g_{i j}(0)=\delta_{i j}, B_{1}^{+}(0) \subset M,\left\{\left(x^{\prime}, 0\right):\left|x^{\prime}\right|<1\right\} \subset \partial M$.

Let $R_{\alpha}=1 /\left(\alpha \mu_{\alpha}\right), h_{\alpha}=g_{i j}\left(\mu_{\alpha} x\right) d x^{i} d x^{j}$ in $B_{10 R_{\alpha}}^{+}(0)$, and

$$
\bar{v}_{\alpha}(x)=\mu_{\alpha}^{(n-2) / 2} u_{\alpha}\left(\mu_{\alpha} x\right) \quad \text { for } \quad x \in B_{10 R_{\alpha}}^{+}(0) .
$$

It follows from (4.6) and (4.3) that $R_{\alpha} \rightarrow \infty$ as $\alpha \rightarrow \infty$, and $\bar{v}_{\alpha}$ satisfies

$$
\begin{cases}-\Delta_{h_{\alpha}} \bar{v}_{\alpha}=\zeta_{\alpha} \bar{v}_{\alpha}^{p-1}-\alpha \mu_{\alpha}^{2} \bar{v}_{\alpha} & \text { in } B_{10 R_{\alpha}}^{+}(0), \\ \frac{\partial_{h_{\alpha}} \bar{v}_{\alpha}}{\partial \nu}=-\alpha \mu_{\alpha} \bar{v}_{\alpha} & \text { on }\left\{\left(x^{\prime}, 0\right):\left|x^{\prime}\right|<10 R_{\alpha}\right\} \\ 0<\bar{v}_{\alpha} \leq \mu_{\alpha}^{(n-2) / 2} u_{\alpha}(0) . & \end{cases}
$$

Clearly,

$$
\left|h_{\alpha}^{i j}(x)-\delta^{i j}\right| \leq C\left|\mu_{\alpha} x\right|, \quad\left|\Gamma_{i j}^{k}(x)\right| \leq C \mu_{\alpha} \text { in } B_{10 R_{\alpha}}^{+}(0),
$$

where $\Gamma_{i j}^{k}$ is the Christoffel symbol of $h_{\alpha}$.

As in section 2, we know

$$
\left\|\bar{v}_{\alpha}-v\right\|_{C^{2}\left(B_{10 R_{\alpha}}^{+}\right)} \rightarrow 0
$$

where $v$ was defined by (2.13).

Also from Proposition 4.1, one can see that

$$
\bar{v}_{\alpha}(x) \leq \frac{C}{1+|x|^{n-2}} \quad \text { for } \quad x \in \overline{B_{10 R_{\alpha}}^{+}}(0) .
$$


In turn, we derive as in section 3 the following estimates of $\left|\nabla \bar{v}_{\alpha}\right|$ and $\left|\nabla^{2} \bar{v}_{\alpha}\right|$ by applying standard elliptic theories.

Lemma 4.2. For all $\alpha \geq 1, x \in B_{R_{\alpha}}^{+}(0)$, we have

$$
\left|\nabla \bar{v}_{\alpha}(x)\right| \leq \frac{C}{1+|x|^{n-1}}, \quad\left|\nabla^{2} \bar{v}_{\alpha}(x)\right| \leq \frac{C}{1+|x|^{n}},
$$

where $\left|\nabla^{2} \bar{v}_{\alpha}\right|=\sum_{i, j=1}^{n}\left|\partial^{2} \bar{v}_{\alpha} / \partial x^{i} \partial x^{j}\right|$, and $C$ is some constant independent of $\alpha$ and $x$. For $n=3$, as $\alpha$ large enough, we also have

$$
\bar{v}_{\alpha}(x) \geq \frac{1}{C(1+|x|)}, \quad \forall x \in \overline{B_{R_{\alpha}^{1 / 4}}^{+}(0)} .
$$

As in section 3 , we can derive from (4.9) the following.

$$
\bar{A}\left(h_{\alpha}, \bar{v}_{\alpha}\right)=-J\left(R_{\alpha}, \bar{v}_{\alpha}\right)-I\left(R_{\alpha}, \bar{v}_{\alpha}\right),
$$

where $J\left(R_{\alpha}, \bar{v}_{\alpha}\right), I\left(R_{\alpha}, \bar{v}_{\alpha}\right)$ are defined by (3.8), (3.9) respectively;

$$
\bar{A}\left(h_{\alpha}, \bar{v}_{\alpha}\right)=A\left(h_{\alpha}, \bar{v}_{\alpha}\right)+\alpha \mu_{\alpha}^{2} \int_{B_{R_{\alpha}}^{+}} \bar{v}_{\alpha}^{2} d V-\frac{\alpha \mu_{\alpha}^{2}}{2} \int_{\Gamma_{2}} \bar{v}_{\alpha}^{2}|x| d S,
$$

$A\left(h_{\alpha}, \bar{v}_{\alpha}\right)$ is defined by $(3.12), \Gamma_{1}$ and $\Gamma_{2}$ are defined in a same way in section 3 .

Therefore we have the following Pohozaev type identity

$$
\begin{aligned}
\alpha \mu_{\alpha} \int_{\Gamma_{1}} \bar{v}_{\alpha}^{2} d S=O\left(\int_{\Gamma_{2}} \bar{v}_{\alpha}^{p}|x| d S+\alpha \mu_{\alpha}^{2} \int_{B_{R_{\alpha}}^{+}} \bar{v}_{\alpha}^{2} d V+\alpha \mu_{\alpha}^{2} \int_{\Gamma_{2}} \bar{v}_{\alpha}^{2}|x| d S\right) \\
+O\left(\int_{B_{R_{\alpha}}^{+}} \mu_{\alpha}|x|^{2}\left|\nabla \bar{v}_{\alpha}\right|\left|\nabla^{2} \bar{v}_{\alpha}\right| d V\right)+O\left(\int_{B_{R_{\alpha}}^{+}} \mu_{\alpha}|x|\left|\nabla \bar{v}_{\alpha}\right|^{2} d V\right) \\
+O\left(\int_{B_{R_{\alpha}}^{+}} \mu_{\alpha}|x| \bar{v}_{\alpha}\left|\nabla^{2} \bar{v}_{\alpha}\right| d V\right)+O\left(\int_{B_{R_{\alpha}}^{+}} \mu_{\alpha} \bar{v}_{\alpha}\left|\nabla \bar{v}_{\alpha}\right| d V\right) \\
+O\left(\int_{\Gamma_{2}}\left(|x|\left|\nabla \bar{v}_{\alpha}\right|^{2}+\bar{v}_{\alpha}\left|\nabla \bar{v}_{\alpha}\right|\right) d S\right) \\
+O\left(\int_{\partial \Gamma_{1}} \alpha \mu_{\alpha} \bar{v}_{\alpha}^{2}|x| d S\right) \\
+O\left(\int_{\Gamma_{1}}\left[\mu_{\alpha}\left|x^{\prime}\right|^{2}\left|\nabla \bar{v}_{\alpha}\right|^{2}+\mu_{\alpha}\left|x^{\prime}\right| \bar{v}_{\alpha}\left|\nabla \bar{v}_{\alpha}\right|\right] d S\right)
\end{aligned}
$$

From (4.11) and Lemma 4.2, we have the following lemma.

LEMma 4.3. For $n \geq 3$, there exists some constant $C>0$ independent of $\alpha$, such that $\int_{\Gamma_{1}} \bar{v}_{\alpha}^{2} d S>1 / C$ for all $\alpha \geq 1$. Moreover for $n=3$, $\int_{\Gamma_{1}} \bar{v}_{\alpha}^{2} d S \geq\left(\log R_{\alpha}\right) / C$ for all $\alpha \geq 1$.

Also, by (4.12), Lemma 4.2 and some elementary calculations, we have 
Lemma 4.4. The following estimates hold.

$$
\begin{aligned}
& \int_{\partial \Gamma_{1}} \alpha \mu_{\alpha} \bar{v}_{\alpha}^{2}|x| d S \leq \alpha \mu_{\alpha} R_{\alpha}^{3-n}, \\
& \alpha \mu_{\alpha}^{2} \int_{\Gamma_{2}} \bar{v}_{\alpha}^{2}|x| d S \leq \frac{1}{\alpha}\left(\alpha \mu_{\alpha}\right)^{n-2}, \\
& \alpha \mu_{\alpha}^{2} \int_{B_{R_{\alpha}}^{+}} \bar{v}_{\alpha}^{2} d V \leq \begin{cases}C \mu_{\alpha}, & n=3 \\
C \alpha \mu_{\alpha}^{2} \log R_{\alpha}, & n=4 \\
C \alpha \mu_{\alpha}^{2}, & n \geq 5\end{cases} \\
& \int_{\Gamma_{1}}\left(\mu_{\alpha}\left|x^{\prime}\right|^{2}\left|\nabla \bar{v}_{\alpha}\right|^{2}+\mu_{\alpha}\left|x^{\prime}\right| \bar{v}_{\alpha}\left|\nabla \bar{v}_{\alpha}\right|\right) d S \begin{cases}C \mu_{\alpha} \log R_{\alpha}, & n=3, \\
C \mu_{\alpha}, & n \geq 4,\end{cases} \\
& \int_{\Gamma_{2}}\left(|x|\left|\nabla \bar{v}_{\alpha}\right|^{2}+\bar{v}_{\alpha}\left|\nabla \bar{v}_{\alpha}\right|\right) d S \leq C\left(\alpha \mu_{\alpha}\right)^{n-2}, \\
& \int_{\Gamma_{2}} \bar{v}_{\alpha}^{p}|x| d S \leq C\left(\alpha \mu_{\alpha}\right)^{n}, \\
& \int_{B_{R_{\alpha}}^{+}}\left(\mu_{\alpha}|x|^{2}\left|\nabla \bar{v}_{\alpha}\right|\left|\nabla^{2} \bar{v}_{\alpha}\right|+\mu_{\alpha}|x|\left|\nabla \bar{v}_{\alpha}\right|^{2}\right) d V \leq \begin{cases}C \mu_{\alpha} \log R_{\alpha}, & n=3, \\
C \mu_{\alpha}, & n \geq 4,\end{cases} \\
& \int_{B_{R_{\alpha}}^{+}}\left(\mu_{\alpha}|x| \bar{v}_{\alpha}\left|\nabla^{2} \bar{v}_{\alpha}\right|+\mu_{\alpha} \bar{v}_{\alpha}\left|\nabla \bar{v}_{\alpha}\right|\right) d V \leq \begin{cases}C \mu_{\alpha} \log R_{\alpha}, & n=3, \\
C \mu_{\alpha}, & n \geq 4,\end{cases}
\end{aligned}
$$

Proof of Theorem 0.2. By Lemma 4.3 and Lemma 4.4, we easily derive a contradiction from (4.14) as $\alpha$ tends to infinity.

\section{References}

[AY] Adimurthi, S.L. Yadava, Some Remarks on Sobolev Type Inequalities, Calc. Var. and PDEs 2 (1994), 427-442.

[Au1] T. Aubin, Espaces de Sobolev sur les variétes Riemanniennes, Bull. Sc. Math. 100 (1976), 149-173.

[Au2] T. Aubin, Nonlinear Analysis on Manifolds, Monge-Ampere Equations, Grundlehern math. Wiss. 252, Springer-Verlag, Berlin, 1982.

[B] W. Beckner, Sharp Sobolev inequalities on the sphere and the MoserTrudinger inequality, Ann. Math. 138 (1993), 213-242.

[BrLi] H. Brezis, E. Lieb, Sobolev inequalities with remainder terms, J. Funct. Anal. 62 (1985), 73-86.

[BrNi] H. Brezis, L. Nirenberg, Positive solutions of nonlinear elliptic equations involving critical Sobolev exponents, Comm. Pure Appl. Math. 36 (1983), 437-477. 
[CGS] L. Caffarelli, B. Gidas, J. Spruck, Asymptotic symmetry and local behavior of semilinear elliptic equations with critical Sobolev growth, Comm. Pure Appl. Math. 42 (1989), 271-297.

[CaLo1] E. Carlen, M. Loss, Extremals of functionals with competing symmetry, J. Funct. Anal. 88 (1990), 437-456.

[CaLo2] E. Carlen, M. Loss, On the minimization of symmetric functionals, Reviews in Mathematical Physics, Special Issue (1994), 1011-1032.

[CarCh] L. Carleson, S.Y.A. Chang, On the existence of an extremal function for an inequality of J. Moser, Bull. Sc. Math. 110 (1986), 113-127.

[Che1] P. Cherrier, Problèmes de Neumann nonlinéaires sur les variétés Riemanniennes, J. Func. Anal. 57 (1984), 154-207.

[Che2] P. Cherrier, Meilleures constantes dans inégalités relatives aux espace de Sobolev, Bull. Sci. Math. 108 (1984), 225-262.

[E1] J. EscobAR, Uniqueness theorems on conformal deformation of metric, Sobolev inequalities, and an eigenvalue estimate, Comm. Pure Appl. Math. 43 (1990), 857-883.

[E2] J. EscobaR, Sharp constant in a Sobolev trace inequality, Indiana Univ. Math. J. 37 (1988), 687-698.

[GNNi] B. Gidas, W.M. Ni, L. Nirenberg, Symmetry and related properties via the maximum principle, Comm. Math. Phys. 68 (1979), 209-243.

[H] H. Hamza, Sur les transformations conformes des variétés Riemanniennes à bord, J. Func. Anal. 92 (1990), 403-447.

[He] E. Hebey, Optimal Sobolev inequalities on complete Riemannian manifolds with Ricci curvature bounded below and positive injectivity radius, American Journal of Mathematics 118 (1996), 291-300.

[HeV1] E. Hebey, M. Vaugon, Meilleures constantes dans le theoreme d'inclusion de Sobolev, Annales de l'Institut Henri Poincare, Analyse nonlineaire 13 (1996), 57-93.

[HeV2] E. Hebey, M. Vaugon, The best constant problem in the Sobolev embedding theorem for complete Riemannian manifolds, Duke Math. J. 79 (1995), 235-279.

[LZ] Y.Y. LI, M. ZHU, Sharp Sobolev trace inequality on Riemannian manifolds with boundary, Comm. Pure Appl. Math. 50 (1997), 449-487.

[Li1] E. LIEB, Existence and uniqueness of the minimizing solution of Choquard's nonlinear equation, Studies in Appl. Math. 57 (1977), 93105.

[Li2] E. LieB, Sharp constants in the Hardy-Littlewood-Sobolev and related inequalities, Ann. of Math. 118 (1983), 349-374.

[Lio] P.L. Lions, The concentration compactness principle in calculus of variations, the limit case, Rev. Mat. Iberoamericana 1 (1985), 145-201.

[M] J. Moser, A sharp form of an inequality by N. Trudinger, Indiana Math. J. 20 (1971), 1077-1092. 
[T] G. Talenti, Best constant in Sobolev inequality, Ann. Mat. Pure Appl. 110 (1976), 353-372.

[Tr] N. Trudinger, On imbedding into Olicz spaces and some applications, J. Math. Mech. 17 (1967), 473-484.

Yan Yan Li and Meijun Zhu Current address of Meijun Zhu

Department of Mathematics

Rutgers University

New Brunswick, NJ 08903

USA

\author{
Dept. of Math. \\ Univ. of British Columbia \\ Vancouver, BC \\ Canada V6T $1 Z 2$
}

Submitted: January 1997 